\title{
Wake of two side-by-side square cylinders at low Reynolds numbers
}

\section{Shengwei Ma ${ }^{1}$ Chang-Wei Kang ${ }^{1}$ Teck-Bin Arthur Lim ${ }^{1}$ Chih-Hua Wu ${ }^{1}$ Owen Tutty ${ }^{2}$}

${ }^{1}$ Institute of High Performance Computing, A*STAR, Singapore

${ }^{2}$ Southampton Marine and Maritime Institute, University of Southampton, Southampton, UK

Abstract: Wake of two side-by-side square cylinders was investigated through direct numerical simulation at low Reynolds numbers (16 200). The gap between the two cylinders varied from $0-10 D$, where $D$ is the dimension of the square cylinder (edge length). 9 different wake patterns and their dependency on both Reynolds number and gap spacing were identified and analysed. A system classification map, demarcated by Reynolds number and gap ratio $g^{*}(g / D$, where $g$ is the gap spacing between 2 cylinders), was derived for these 9 wake modes. Steady-state wake (mode I) was observed when Reynolds number is lower than the critical Reynolds number, which depends on $\mathrm{g}^{*}$. For gap ratio less than 0.7 , only single vortex street was observed. The single vortex street wake can be either symmetric and periodic (mode II), or asymmetric and periodic (mode III), or irregular (mode IV). In this gap ratio range (less than 0.7 ), shedding frequency decreases with gap ratio due to the damping role of the gap flow. For gap ratio larger than 0.7 , two vortex streets were also observed. For gap ratio larger than 1, only two vortex streets were observed. Vortex shedding can be either synchronized and in-phase (mode $\mathrm{V}$ ), synchronized and anti-phase (mode $\mathrm{VI}$ ), in-phase dominated with low frequency modulation (mode VII), anti-phase dominated with low frequency modulation (mode VIII), asymmetric synchronized anti-phase (mode IX), or irregular (mode IV). For gap ratio larger than 4, only synchronized anti-phase mode was observed under the conditions of this study. In the two vortex streets regime, shedding frequency is higher than that of a single cylinder, due to stronger gap flow than that in the freestream side. The impact of gap ratio and Reynolds number on the drag and lift forces was also studied. 


\section{Introduction}

Fluid flow around slender bluff bodies plays an important role in many engineering applications, e.g., flow control, structural design, vibration control, aeroelastic instability and flow-induced vibration. One of the key phenomena in such engineering systems is vortex shedding which results in periodic or quasi-periodic loading on the bluff body. Therefore, many studies have been conducted towards understanding the phenomenon (Roshko, 1961; Gerrard, 1966; King, 1977; Norberg, 1993; Williamson, 1996; Sumer and Fredsøe, 2006). For a single cylinder, stable periodic vortex shedding usually can be observed when the Reynolds number $\left(\operatorname{Re}=U_{0} D / v\right.$, where $\mathrm{U}_{0}$ is the freestream velocity or inlet velocity, $v$ kinematic viscosity of the fluid, and $D$ the diameter of the cylinder) reaches about $40-50$ depending on blockage and other experimental conditions. Increasing the Reynolds number will gradually elevate the shedding frequency, which is usually represented by the Strouhal number ( $S_{t}=f D / U_{0}$, where $f$ is the shedding frequency). When Re reaches about 170 225 (depending on experimental conditions), $2 \mathrm{D}$ to $3 \mathrm{D}$ flow transition will usually be found to happen. Up to $\operatorname{Re}$ around $200 \sim 300$, the flow is still laminar; but vortex shedding and the wake become three-dimensional. Turbulent wake may be observed if $R e$ is further increased (Williamson, 1996).

When two cylinders are placed side-by-side, the flow characteristics could be substantially different from those of single cylinder due to interactions of the wakes of the two cylinders. Many researchers suggested that the flow behind two side-by-side cylinders is mainly governed by the two non-dimensional parameters of Reynolds number $(\mathrm{Re})$ and gap ratio, $\mathrm{g}^{*}$ $(g / D)$, where $g$ is the spacing between 2 cylinders (Williamson, 1985; Kang, 2003; Mizushima and Ino, 2008; Carini et al, 2014). For square cylinders, the length of one edge is usually used as characteristic length $\mathrm{D}$ for both $\mathrm{Re}$ and $\mathrm{g}^{*}$. In last few decades, the wake of two side-by-side circular cylinders has been extensively studied through both experiments and numerical simulations. Understanding of the wake patterns, the interference of the two 
cylinders at low gap ratios and wake interactions has been advanced gradually. For example, Bearman and Wadcock (1973) investigated the role of $\mathrm{g}^{*}$ in interaction of the two cylinders at $\operatorname{Re}=25,000$ experimentally. They found that for $\mathrm{g}^{*}$ between 0.1 and 1 , the wake was asymmetric. Such an asymmetry was found to be more pronounced when the cylinders were replaced with a pair of two-dimensional flat plates. Therefore, they concluded that it was unlikely that the asymmetric flow was the result of the boundary-layer effect. When $g^{*}>1$, vortex shedding was synchronized and in-phase, and two vortex streets were observed. Williamson (1985) studied vortex shedding at low Reynolds numbers through flow visualization. Synchronized vortex shedding was observed at $1<\mathrm{g}^{*}<5$. Both in-phase and anti-phase shedding were captured with the dominance of anti-phase mode in this gap ratio range. For in-phase shedding mode, single binary-vortex street comprised of combined pairs of like-signed vortices was found to be formed downstream, instead of two parallel vortex streets as that in anti-phase shedding mode. For $\mathrm{g}^{*}<1$, asymmetric wake, having specific harmonic vortex shedding modes, was observed. With $0 \leq g^{*} \leq 5$ and $500 \leq \operatorname{Re} \leq 3000$ in a water tunnel and a towing tank, Sumner et al (1999) observed 3 basic flow patterns: single vortex street $\left(g^{*}<0.2\right)$, biased (asymmetric) flow with synchronized shedding $\left(0.2<\mathrm{g}^{*}<1.2\right)$ and symmetric flow with synchronized vortex shedding. Kang (2003) studied the impact of gap ratio $\left(g^{*} \leq 5\right)$ and $\operatorname{Re}(40-160)$ on the wake of two side-by-side circular cylinders. Six different wake patterns, steady-state, single bluff-body, deflected (asymmetric), flip-flopping, synchronized in-phase, and synchronized anti-phase, were identified through numerical simulations. The results suggested that at low Reynolds numbers, the interaction of the two cylinders could be more complicated than that at higher Reynolds numbers. The simulation results also confirmed that in-phase vortex shedding leads to the development of single binary-vortex street in the downstream reported by Williamson (1985) in experiment. Wang and Zhou (2005) investigated experimentally the wake pattern and role of gap flow in the wake of a pair of cylinders for three gap ratios $(0.13,0.7$ and 2$)$ with $R e=120--1100$ (PIV and hot-wire measurements were conducted at Re 5900). They found that the asymmetric wake $\left(g^{*}=0.7\right)$ and the changeover of deflection direction was very likely linked to the interaction 
among the gap vortices. However, Carini et al (2014) gave a different explanation on the origin of the flip-flop instability (i.e., the changeover of two asymmetric states). Based on Floquet analysis, they concluded that the flip-over was originated from the unstable mode of the in-phase synchronized vortex shedding that was observed to happen before the flip-flop state was developed in their DNS simulations for $0.6<\mathrm{g}^{*}<1.4$ and $50<\operatorname{Re} \leq 90$. ..

For the wake of two side-by-side square cylinders, relatively fewer researches have been reported. Kolár et al (1997) investigated experimentally the near wake features of two sideby-side square cylinders with $g^{*}=2$ and $R e=23100$. They found that vortical structures originated from the gap decayed dramatically faster than those from outer (freestream) side. A coupled vortex street predominantly in anti-phase mode was observed in the experiment. Yen and Liu (2011) studied the wake flow of two side-by-side square cylinders in a wind tunnel with $2262<\operatorname{Re}<28,000$ and $0 \leq g^{*} \leq 12$. They classified the wake into 3 modes: single mode $\left(g^{*} \leq 0.1\right)$, gap-flow mode with anti-phase vortex shedding $\left(0.1 \leq g^{*} \leq 5.5\right)$ and coupled inphase vortex-shedding mode $\left(g^{*} \geq 5.5\right)$. Alam et al (2011) did a series of experiments with different gap ratios varying from 0.2 to 5 at $R e=47000$ and $R e=17000$ to investigate the wake flow of 2 side-by-side square cylinders systematically. They identified 4 distinct flow regimes, comprising of (i) single-body regime for $\mathrm{g}^{*}<0.3$; (ii) two-frequency regime with a narrow and a wide wake for $0.3<g^{*}<1.2$; (iii) transition regime for $1.2<g^{*}<2$; and (iv) coupled vortex street regime for $\mathrm{g}^{*}>2$ (with anti-phase dominated vortex shedding in $2<\mathrm{g}^{*}<3.6$, and anti-phase and in-phase mixed in $\left.3.6<\mathrm{g}^{*}<5\right)$. They also compared the results with those of circular cylinders and suggested that wakes behind 2 side-by-side square cylinders may differ from circular cylinders at same $\mathrm{g}^{*}$ and $\mathrm{Re}$ due to the differences in geometry of the gap and flow separation locations. Alam and Zhou (2013), through flow visualization, found similar 4 regimes at a far lower Reynolds number of 300 with similar $\mathrm{g}^{*}$ ranges: single bluff body regime for $\mathrm{g}^{*}<0.2$; narrow and wide streets regime for $0.2<\mathrm{g}^{*}<1.1$; transition regime for $1.1<\mathrm{g}^{*}<1.4$; and coupled regime for $\mathrm{g}^{*}>1.4$. Burattini and Agrawal (2013) simulated wake interactions of 2 side-by-side cylinders at $\operatorname{Re}=73$ and $0.5 \leq g^{*} \leq 6$. Four wake flow regimes: 
single vortex street, quasi-periodic, in-phase and anti-phase, were reported depending on $\mathrm{g}^{*}$.

But the range of $\mathrm{g}^{*}$ corresponding to different regimes were not systematically reported.

Their results were consistent with their earlier calculations using a similar numerical model (Agrawal et al 2006).

As suggested by previous researchers, the wake flow regimes are mainly determined by both Reynolds number and gap ratio at low Reynolds numbers. These previous researches were however mostly focused on studies of gap ratio effects at few relatively high Reynolds numbers regime. Therefore, the wake patterns of two side-by-side square cylinders at low Reynolds numbers and their transitions have not been studied much and thus, still not well understood. For example, how will the wake pattern and vortex shedding mode change systematically with $\mathrm{g}^{*}$ and $\mathrm{Re}$ at low Reynolds numbers? How will the shedding frequency and force coefficients? What's the role of the gap flow in the wake patterns and vortex shedding and how does its role change with $\operatorname{Re}$ and $g^{\star}$ ?

The purpose of this study is to systematically investigate the wake flow of 2 side-by-side square cylinders at low Reynolds numbers to advance the understanding of wake flow regimes and the role of gap flow in the transitions of wake patterns. In this paper, the discussion is limited to $2 \mathrm{D}$ laminar flow regime with $\mathrm{Re} \leq 200$. Some stability analyses and experimental studies indicated that the flow actually would be transitioned to $3 D$ when $R e$ is around 170 for a single cylinder (Noack and Eckelmann, 1994; Williamson, 1995; Barkley and Henderson, 1996; Robichaux et al 1999; Luo et al, 2006; Choi and Yang, 2013). This subcritical Reynolds number may decrease with $\mathrm{g}^{*}$ for two side-by-side cylinders. But for $R e<200$, the flow in spanwise direction is still developing and the 2D features of the flow may not be altered significantly. As such, the assumption of $2 \mathrm{D}$ flow and hence using $2 \mathrm{D}$ simulation for this problem can generally be still valid. 


\section{Problem setup and numerical method}

The computing domain is illustrated in Figure 1. Two identical square cylinders with a dimension of $D$ are placed symmetrically in y direction in the domain. The distance (gap) between two cylinders, $g$, varies from $0-10 D$ and in total 30 gap ratios $\left(\mathrm{g}^{*}\right)$ were used in the calculations. They are $0,0.1,0.2,0.3,0.35,0.4,0.5,0.6,0.7,0.8,0.9,1,1.2,1.5,1.6,1.8,2$, $2.2,2.5,2.8,3,3.2,3.5,4,5,6,7,8,9$, and 10 . The computing domain is $51 \mathrm{D}$ in length $(\mathrm{x})$ and $30 \mathrm{D}$ in height $(\mathrm{y})$. The origin $(0,0)$ is set at the center of the gap.

Assuming the fluid is incompressible and Newtonian, fluid flow inside the domain can then be modelled with Navier-Stokes equations:

$$
\begin{gathered}
\nabla \cdot \mathbf{u}=0 \\
\frac{\partial \mathbf{u}}{\partial t}+(\mathbf{u} \cdot \nabla) \mathbf{u}=\nabla p+v \nabla^{2} \mathbf{u}
\end{gathered}
$$

where $\mathbf{u}$ is the velocity vector field with $u$ and $v$ as components in $\mathrm{x}$ and $\mathrm{y}$ directions respectively, $p$ the pressure and $v$ the kinematic viscosity.

At the inlet boundary $(x=-9.5 D)$ constant velocity was assumed $\left(u=U_{0}, v=0\right)$. On the cylinder surface, non-slip boundary $(u=0, v=0)$ was used. Outflow boundary $(\partial u / \partial x=0, \partial v / \partial x=0)$ was assumed in the outlet $(x=41.5 \mathrm{D})$. Symmetry boundary condition $(\partial u / \partial y=0, v=0)$ was used in the top and bottom boundaries $(y= \pm 15 D)$. A zero gradient pressure boundary was assumed in all boundaries, except at the inlet, where reference pressure was set as 0 . Initial conditions in the domain were set as $u=0, v=0$ and $p=0$ for all cases.

With the boundary conditions and initial conditions, the governing equations (equations 1 and 2) were then solved numerically using OpenFOAM® (Open Field Operation and Manipulation) CFD toolbox, which is a collection of C++ libraries developed for solving continuum mechanics problems with finite volume method (Weller et al, 1998). The pimpleFoam solver in OpenFOAM, which is a transient solver for incompressible Newtonian fluid flow using the PIMPLE algorithm that combines SIMPLE (Semi-Implicit Method for Pressure-Linked Equations) and PISO (Pressure Implicit with Splitting of Operator) algorithm 
for pressure-velocity coupling (Moukalled at al, 2016), $2^{\text {nd }}$ order spatial (Gauss linear) and $2^{\text {nd }}$ order temporal (Crank-Nicholson) schemes, were used in this study. Time-step size is fixed at $0.0025 T_{0}\left(T_{0}=D / U_{0}\right)$ for all cases. So, the maximum Courant number in most cases is less than 0.5 . The calculations were run until the periodic flow field is statistically converged $\left(\frac{d}{d t}\left(\frac{\int_{t_{0}}^{t} u d t}{t-t_{0}}\right)<10^{-10} ; \frac{d}{d t}\left(\frac{\int_{t_{0}}^{t} v d t}{t-t_{0}}\right)<10^{-10} ; \frac{d}{d t}\left(\frac{\int_{t_{0}}^{t} p d t}{t-t_{0}}\right)<10^{-10}\right.$, where $t_{0}$ is the start time when flow is apparently periodical). Solutions were monitored at about 100 points mainly in the wake and around the cylinders. If the flow is irregular, calculation run until drag coefficient and lift coefficient were statistically converged $\left(\frac{d}{d t}\left(\frac{\int_{0}^{t} C_{D} d t}{t}\right)<10^{-10} ; \frac{d}{d t}\left(\frac{\int_{0}^{t} C_{L} d t}{t}\right)<\right.$ $10^{-10}$, where $C_{D}$ is the drag coefficient for each cylinder and $C_{L}$ the lift coefficient). The typical simulated duration is in the order of $10^{3} \mathrm{~T}_{0}$.

To solve the equations numerically, the computing domain was meshed with non-uniform structured mesh using ANSYS ICEM CFD. Each edge of the cylinder was divided into 25 mesh elements based on mesh convergence study. In regions around each cylinder except the gap side (4D in $+x$ direction and $2 \mathrm{D}$ in other 2 directions), mesh nodes were placed with a hyperbolic distribution. The height of the first layer mesh around cylinder was fixed at 0.01D. The maximum growth rate of mesh is about $1 \%$ in downstream of the two cylinders ( $+x$ direction) and about $2.5 \%$ in other 2 directions (upstream and freestream side of the cylinders). Outside this region, the mesh growth rate is about $1 \%$. The maximum mesh size in $x$ direction (at $x=41.5 \mathrm{D}$ ) is $0.15 \mathrm{D}$ and $0.2 \mathrm{D}$ (at $\mathrm{y}= \pm 15 \mathrm{D}$ ) in $\mathrm{y}$ direction. Within the gap, the meshing is treated specially to ensure good mesh fidelity. For $\mathrm{g}^{*} \leq 0.5$, uniform layers (with a thickness of $0.01 \mathrm{D})$ were placed. For $\mathrm{g}^{*}>0.5$, the mesh nodes were placed with a hyperbolic distribution. The maximum growth rate is restricted to $2 \%$ and maximum thickness varies from $0.02 \mathrm{D}$ to $0.05 \mathrm{D}$ depending on gap ratio. Using these distributions, the number of total quad elements was found to vary from 193694 to 362882 depending on $\mathrm{g}^{*}$. Part of the mesh for $\mathrm{g}^{*}=1$ used in the simulation is shown in Figure 2 . 
In addition to OpenFOAM calculations for the flow field, linear stability analysis was conducted to determine the onset of vortex shedding using Nektar++, which is an opensource software framework using the spectral/hp element method to solve partial differential equations (Cantwell et al, 2015). Direct stability analysis tools in Nektar++ were used in this study with an identical domain as shown in Figure 1. In linear stability analysis, a steadystate base flow solution for each $\mathrm{g}^{*}$ and $\mathrm{Re}$ was obtained from Nektar++ using adaptive selective frequency damping method (Jordi et al, 2015) with polynomial order of 8 first. Linear stability analysis was then conducted for this base flow with the same polynomial order (Cantwell and Barkley, 2010).

\section{Convergence check and validation}

\section{A. Mesh convergence check}

To get mesh-independent solutions, it is important to get sufficient mesh resolution near the cylinder and in the wake. For example, for the cylinders, the number of divisions in each side (i.e., mesh size on the cylinder surface) is equally important as the first layer mesh thickness. As shown in Figure 3, different mesh sizes varying from 0.1D (10 divisions in each side) to $1 / 30 \mathrm{D}$ on cylinder surface were tested for the case with one square cylinder at $R e=100$. It is found that the solution converged as the surface mesh size is refined to around 0.04D 0.05D (with 0.01D for the first layer mesh thickness). For example, when decreasing cylinder surface mesh size from $1 / 10 \mathrm{D}$ to $1 / 20 \mathrm{D}$, Strouhal number increased about $1.1 \%$ and the mean drag coefficient decreased about $1.04 \%$. But when decreasing mesh size on cylinder surface from $1 / 25 \mathrm{D}$ to $1 / 30 \mathrm{D}$, the change in Strouhal number was less than $0.5 \%$ and change in mean drag coefficient was almost negligible. Similarly, it is found that a cylinder wall first layer mesh thickness refinement of around 0.01D is sufficient to get the mesh-independent solutions at the studied Reynolds number range. These requirements are comparable to meshing requirements that were reported by Sohankar et al (1999) for flow across a square cylinder problem with Reynolds number up to 500 . 


\section{B. Comparison to experimental results for a single cylinder case}

Due to limited availability of quantitative experimental data on 2 side-by-side square cylinders at low Reynolds numbers (less than 200), a testing case with one square cylinder was used to benchmark our numerical solutions on the general trends of the key parameters in vortex shedding including Strouhal number and mean drag coefficient. In our calculations on a single square cylinder, the channel height was taken as 18 , giving a blockage ratio of $5.56 \%$.

Figure 4 shows the comparison of current results with the experimental data reported in literature (Okajima, 1982; Okajima, 1995; Norberg, 1996, reported in Sohankar et al, 1997), and the numerical solutions of Franke et al (1990) and Sohankar et al (1995). It is found in the studied Reynolds number range, our simulations can capture the similar trends for Strouhal number and mean drag coefficient as with the others reported. For Strouhal number (Figure 4(a)), our simulation results, which are close to Sohankar's, and Franke's simulations are noticeably higher than the experimental data. This is very likely mainly due to the lower blockage ratio in experiments. In the water tank experiment (Okajima 1982), the blockage ratio is about $2 \%$. In Norberg's experiment, the blockage ratio is about $0.25 \%$ (Sohankar et al, 1997). Both are significantly lower than that in our simulations (5.56\%) and Franke's $(8.33 \%)$. In the work of Sohankar et al (1995), a blockage ratio of $5 \%$ was used, which is close to ours, therefore the Strouhal numbers from our simulations are very close to theirs up to Reynolds number of 150.

For the mean drag coefficient (Figure 4(b)), comparable and consistent trends can be observed from all simulations. But there is a systematic shift of the absolute values of the mean drag coefficient at all Reynolds numbers among 3 sets of simulation results. Our results are consistently $6.2 \% \sim 11.5 \%$ lower than Franke's, yet consistently $2.65 \% \sim 4.75 \%$ higher than Shankar's at the same Reynolds number. Such differences among three sets of simulation results of the mean drag coefficient may be apparently due to the difference in 
blockage ratio employed and numerical schemes used. The differences between simulations and the experiments is probably due to differences in blockage ratio, experimental conditions and uncertainties in experiments.

\section{The impact of $\mathbf{g}^{*}$ and $\mathrm{Re}$ on wake patterns for 2 side-by-side square cylinders}

In this study, we were interested in the final state (periodic or irregular) of the wake patterns, From our simulation results of the 2 side-by-side square cylinders obtained, 9 different wake patterns/modes were identified for the conditions of $g^{*}$ varying from 0-10 and $\operatorname{Re}$ up to 200 . The ranges of $\mathrm{g}^{*}$ and $\mathrm{Re}$ of each mode were plotted in Figure 5. These 9 modes/patterns as shown in Figure 5 are: steady-state wake (mode I), symmetric single vortex street (mode II), asymmetric single vortex street (mode III), irregular vortex shedding (mode IV), synchronized in-phase vortex shedding (mode V), synchronized anti-phase vortex shedding (mode VI), synchronized in-phase dominated vortex shedding with low frequency modulation (mode VII), synchronized anti-phase dominated vortex shedding with low frequency modulation (mode VIII), and asymmetric synchronized anti-phase vortex shedding (mode IX). In this plot, the resolution of the Reynolds number studied in our simulations is about 10 for $\operatorname{Re}>100,5$ for $100>\operatorname{Re}>60$, and usually less than 2.5 for $\operatorname{Re}<60$. The resolution of the gap ratio studied is in accordance to the 30 values, set out in Section II. The boundary lines among different modes were obtained from linear interpolation of $\operatorname{Re}$ and $\mathrm{g}^{*}$ from the nearest points, except for the boundary of mode I, whose boundary was obtained from linear stability analysis discussed later. For example, at $\mathrm{g}^{*}=3$, for $43 \leq \operatorname{Re} \leq 46$, OpenFOAM simulation results indicated the wake pattern was mode IX, mode VIII for $47 \leq \operatorname{Re} \leq 52$, mode $V$ for $53 \leq \operatorname{Re} \leq 72.5$, and mode $\mathrm{VI}$ for $\mathrm{Re} \geq 75$. So, at $\mathrm{g}^{*}=3$, the boundary between mode IX and mode VIII was determined as $\mathrm{Re}=46.5$. Similarly, $\mathrm{Re}=52.5$ was determined as boundary between mode $\mathrm{VIII}$ and mode $\mathrm{V}$, and $\mathrm{Re}=73.75$ as the boundary between mode $\mathrm{V}$ and Mode $\mathrm{VI}$ at $\mathrm{g}^{*}=3$. The same type of boundary points were then connected linearly to form the boundary among these modes. We readily acknowledge that the blockage ratio and entrance length in the computing domain may cause some slight changes on the influence of 
$\operatorname{Re}$ and $\mathrm{g}^{*}$ on the 9 wake patterns/modes. The impact of these parameters and other factors will be discussed separately in another paper.

Within the region of synchronized anti-phase vortex shedding mode regime (mode VI), there is a noticeable sub-region (shaded area in Figure 5), whereby the in-phase shedding mode (mode V) could be easily obtained with certain initial perturbations at the start of the simulations. In other words, if no initial perturbation is added, the final state would be the anti-phase shedding mode (mode VI); and conversely, if certain initial perturbation is added (then removed after a few periods), the final state would be the in-phase shedding mode (mode V). Such similar bi-stable shedding modes were reported too in experiments for circular cylinders at similar gap ratios (Williamson, 1985). In his experiments, both stable inphase and anti-phase shedding modes were observed at $g^{*}=3$ and $\operatorname{Re}=100$. There are probably different ways to trigger such initial condition-dependent wake patterns in this range of $\operatorname{Re}$ and $g^{*}$. In our study, during the first 100 dimensionless time units of $T_{0}\left(D / U_{0}\right)$, a sinusoidal perturbation for $y$ component velocity was added in the inlet boundary to get the in-phase shedding mode as the final state. The systematic way to trigger in-phase mode in this region and other possible initial condition-dependent shedding modes will be discussed separately in another paper. In this paper, subsequent discussion is based on cases of initial conditions of $u=y=p=0$ without any perturbations.

The 9 wake patterns and vortex shedding modes were derived and identified based on analyses using several criteria of: (1) visualization of vortex shedding (Figure 6 shows instantaneous vorticity field for different modes); (2) existence of stationary shedding frequency (Figure 7 shows 3 types of possible results from fast Fourier transformation (FFT) analysis of instantaneous lift and drag coefficients); (3) instantaneous and mean drag coefficient (Figure 8 shows instantaneous drag coefficient of two cylinders); (4) instantaneous and mean lift coefficient (Figure 9 shows instantaneous lift coefficient of two cylinders); (5) instantaneous flow field (Figure 10 shows instantaneous $u$ and $v$ in two points and their correlations); (6) mean flow field (Figure 11 shows mean flow field visualization and 
the corresponding recirculation regions); (7) pressure fluctuations (Figure 12 shows the spatial distribution of $\overline{p^{\prime 2}} /\left(\rho^{2} U_{0}^{4}\right) ;(8)$ x-component velocity fluctuations (Figure 13 shows the spatial distribution of $\overline{u^{\prime 2}} / U_{0}^{2}$ ), and (9) y-component velocity fluctuations (Figure 14 shows the spatial distribution of $\overline{v^{\prime 2}} / U_{0}^{2}$ ). The features of these criteria under different modes will be discussed later.

The 9 wake modes identified are comparable to those reported by Kang (2003) for 2 side-byside circular cylinders at low Reynolds numbers that gave 6 wake modes in their study. Here, for our square cylinders study, we observed 3 additional modes, i.e., the in-phase dominated shedding mode with low frequency modulation (mode VII), the anti-phase dominated shedding mode with low frequency modulation (mode VIII), and the asymmetric anti-phase mode (mode IX). The corresponding Re-g* ranges for other 6 wake modes were noticeably different. But the transition path from one mode to another among these 6 modes were similar when changing $\operatorname{Re}$ and $\mathrm{g}^{*}$. The 3 new modes apparently added additional transition paths for other 6 modes. And additionally, when compared to experimental results of two side-by-side square cylinders at a higher $\mathrm{Re}=300$ (Alam and Zhou, 2013), we found and identified 4 new modes at the lower Reynolds numbers regime in this study, namely, mode III (asymmetric single vortex street mode) at Re up to 125; mode VII for Re up to 65, mode VIII for Re up to 57.5 and mode IX for Re up to 49. The narrow-and-wide street regime, and transition regime reported in their experimental results are probably corresponding to irregular mode (mode IV) in our classifications.

\section{A. Steady-state wake (mode I)}

When Reynolds number is low, the wake flow behind the two square cylinders will be eventually established to a steady-state wake flow irrespective of initial conditions or perturbations. As shown in Figure 6(a), the wake is symmetric about the gap centerline in y direction $(y / D=0)$. A pair of elongated recirculation bubbles can be observed in the downstream (Figure 11(a)). When Reynolds number increases, the recirculation bubbles in 
the downstream of the cylinder will grow until eventually, some unstable modes develops and dominates over the steady mode. The flow then becomes transient and vortex shedding will then be observed. To determine the critical Reynolds number of the onset of the transient flow, linear stability analysis were carried out with Nektar++ based on steady-state base flow which was obtained with Nektar++ by solving steady-state Navier Stokes equations using the boundary conditions mentioned in Section II. The flow would be unsteady when there exist one or more unstable modes with a positive growth rate in the linear stability solutions. At each gap ratio, linear stability analysis was conducted from low Reynolds numbers $\left(\operatorname{Re}=16\right.$ for $g^{*} \leq 1.2$ and $R e=30$ for $g^{*} \geq 1.5$ ). If the flow is steady, i.e., all eigenmodes have negative growth rate, analysis was then conducted on a higher Reynolds number until at least one of the modes has a positive growth rate. Linear stability analysis was then conducted at more Reynolds number until changing of the largest growth rate from negative to positive could be detected with in one (1) Reynolds number range. The critical Reynolds number at each gap ratio, which corresponds to neutral stability with the largest growth rate of 0 , was identified by linear interpolation of the two growth rates indicating the flow regime change. After a critical Reynolds number was identified for a given gap ratio, OpenFOAM simulations were carried out around this critical Reynolds number to verify that final state of the flow is steady-state or transient. The variations of critical Reynolds numbers with different gap ratios are shown in Figure 15.

As indicated in Figure 15, at low gap ratios $\left(g^{*}<0.7\right)$, the critical Reynolds number is close to, yet consistently, lower than that of $g^{*}=0$, which is about 18 . This suggests that the gap flow between two cylinders increases the instability of the system. When gap ratio is larger than 1, the critical Reynolds number is close to that of a single cylinder. This suggests a possible shift of flow physics at $0.7<g^{*}<1$. As shown in Figure 5 , the wake actually changes from single vortex street to two vortex streets in this $\mathrm{g}^{*}$ range. It was also observed that the critical Reynolds number at $1 \leq \mathrm{g}^{*}<10$ are slightly but consistently lower than that of a single cylinder. Again, this suggests the gap increases the instability of the flow and therefore Hopf 
bifurcation occurs at slightly lower Reynolds numbers than that of a single square cylinder.

When $\mathrm{g}^{*}$ increases to 10 , the gap flow may have insignificant impact on the instability of the flow and therefore the critical Reynolds number is similar to that of a single cylinder.

\section{B. Symmetric single vortex street mode (mode II)}

When gap ratio is less than 0.3 , the wake of the two side-by-side cylinders may look similar to the wake of a single cylinder. As shown in Figure 6(b), a single vortex street is formed in the downstream around the centerline in y direction. This indicates vortex shedding only occurs in the outer freestream side, not from the gap side. This mode can be observed at gap ratio up to 0.7 at low Reynolds numbers. For $\mathrm{g}^{*}$ larger than 0.7 , the vortex shedding from the gap side may lead to two vortex streets in the near wake region. Increasing Reynolds numbers at $0.35<\mathrm{g}^{*}<0.8$ will cause the vortex street be biased towards one side of the cylinder, and lead to asymmetric single vortex street mode (mode III) discussed below.

In symmetric single vortex street mode, a stationary shedding frequency is always observed (similar to Figure 7(c)). This frequency is apparently not affected by initial conditions or initial perturbations. The drag coefficients (Figure 8(a)) of the two cylinders have a phase difference of half of the shedding period, while the lift coefficient of the two cylinders is in phase (Figure 9(a)) because vortex shedding occurs in the freestream sides only. Periodic instantaneous velocity field can be observed as shown in Figures 10(c) and 10(d). The correlations of $u$ (Figure 10(a)) and $v($ Figure $10(\mathrm{~b}))$ in two points $(1 \mathrm{D}, 0)$ and $(1.5 \mathrm{D}, 0)$ in the near wake also clearly indicate the flow is periodic and the period for $u$ fluctuations is half of that for $v$.

As shown in Figure 11(c), in this mode (mode II), the mean flow field features a pair of recirculation bubbles in the downstream in the centerline, in addition to the recirculation bubbles immediately behind the cylinders. This suggests that the gap flow is effectively confined by the vortices shed from the freestream side in this mode. Compared to the mean flow field without a gap $\left(g^{*}=0\right.$, Figure $\left.11(b)\right)$, it can be deduced that the gap flow pushed the 
recirculation bubbles, which are associated with vortex shedding from freestream side, downstream in this mode. The recirculation bubbles immediately behind the cylinders are mainly associated with the gap flow due to the sudden expansion of the geometry (gap).

The fluctuations of pressure (Figure 12(a)) clearly indicate single vortex street in the wake. Regions with relatively large pressure fluctuations usually are limited to short distance in the near wake region. Near the centerline $(y / D=0)$, there is a small isolated region with very small pressure fluctuations immediately behind the gap. This, as discussed above, suggests that the gap flow is confined by the vortices shed from freestream side. In mode II, the maximum value of pressure fluctuations is generally larger than that in other modes at the same Reynolds number. The fluctuations of x-component velocity (Figure 13(a)) have similar distribution as pressure fluctuations. The fluctuations of y-component velocity (Figure 14(a)) also indicate single vortex street with maximum fluctuations in the centerline. The maximum value of $\overline{v^{\prime 2}} / U_{0}^{2}$ is larger than that of $\overline{u^{\prime 2}} / U_{0}^{2}$. Compared to corresponding values in the other 7 vortex shedding modes, the maximum value of $\overline{v^{\prime 2}} / U_{0}^{2}$ is the largest for this case of symmetric single vortex street mode (mode II) at the same Reynolds number.

\section{Asymmetric single vortex street mode (mode III)}

At gap ratio between 0.35 and 0.6 , when Reynolds number is gradually increased, the flow will change from steady-state (mode I) to symmetric single vortex street mode (mode II) first. Further increasing the Reynolds number may shift the symmetric vortex street in y direction and forms a biased (towards one cylinder) single vortex street. The instantaneous vorticity of asymmetric vortex street mode is shown in Figure 6(c). Because of the asymmetricity, the wake behind one cylinder is noticeably wider than the wake behind the other cylinder. Once the wide and narrow wake are formed, they will not swap and the flow is then periodic.

In this mode (mode III), the shedding frequency is similar to the symmetric one (single stationary frequency as shown in Figure 7(c)). The frequency of lift coefficient (and drag coefficient) of the two cylinders are identical. But drag coefficient of the two cylinders can be 
noticeably different (Figure 8(b)) and time-averaged net lift force is not 0 (Figure 9(b)). For example, at $\mathrm{g}^{*}=0.4$ and $\mathrm{Re}=80$, cylinder 1 has a mean drag coefficient of 2.04 (with a standard deviation of 0.0159 ); while cylinder 2 has a mean drag coefficient of 2.09 (with a standard deviation of 0.0128 ). The mean lift coefficient for cylinder 1 is 1.09 (with a standard deviation of 0.0144 ), and -1.1413 (with a standard deviation of 0.0204 ) for cylinder 2 . This means the net lift force is downwards in this case. The instantaneous velocity in the wake as shown in Figure 10(g) and Figure 10(h) are periodic. The periodicity can also be verified by the correlation plot of $u$ (Figure 10(e)) and $v$ (Figure 10(f)) in two points. Different from the symmetric case (mode II), the period of $u$ and $v$ fluctuations are identical. Values of $v$ at these two points are all negative. This suggests that the flow is always deflected to cylinder 2 at these 2 points in the near wake region in this case and therefore resulting in a wide wake behind cylinder 1 and a narrow wake behind cylinder 2 . This is consistent with the observations of instantaneous lift coefficients of the two cylinders, described earlier. In the literature, some researchers reported the shedding frequencies were different for the narrow and wide wakes. For example, Williamson (1985) reported the existence of harmonic vortex shedding modes behind a pair of circular cylinders with $g^{*}<1$ and $\operatorname{Re} 200$. For square cylinders, Alam et al (2011) reported a two-frequency regime (higher frequency for the narrow wake and lower frequency for the wide wake) at $0.3<g^{*}<1.2$ with $\operatorname{Re}=4.7 \times 10^{4}$. Very likely, the flow regime in these experiments actually corresponds to irregular shedding mode based on our classification because asymmetric single vortex shedding mode can only be observed for Reynolds number lower than 125. Both experiments reported random swapping of the wide and narrow wake behind the two cylinders. In our simulations, this was observed in irregular shedding mode (Mode IV) discussed below and it is impossible to get periodic flow within a few thousands of dimension time units of $T_{0}\left(D / U_{0}\right)$ when such swapping happens.

In this mode (mode III), the mean flow field is asymmetric about the centerline $(y=0)$ and deflected towards one of the cylinders. In some cases, it is deflected to cylinder 1 , and to 
cylinder 2 in other cases. Apparently, the direction of this deflection is randomly triggered by numerical errors. For example, as shown in Figure 11(d), the recirculation bubble behind cylinder 1 are considerably larger than that behind cylinder 2. In addition, the downstream bubbles are asymmetric as well. The fluctuations of pressure (Figure 12(b)), fluctuations of x-component velocity (Figure 13(b)) and fluctuations of y-component velocity (Figure 14(b)) are all asymmetric. The cause of the asymmetricity is very likely linked to the non-oscillatory mode developed immediately behind the gap. Mizushima and Ino (2008) reported the existence of such a non-oscillatory (stationary) mode (together with other modes) of instability in the wake of two side-by-side circular cylinders at $0.3 \leq \mathrm{g}^{*}<1$ and $\operatorname{Re}>54.2$, based on linear stability analysis. The corresponding pitchfork bifurcation may cause the flow be biased to either side (cylinder 1 or cylinder 2). In numerical calculations, the numerical errors may trigger the flow to be biased to cylinder 1 or cylinder 2 randomly due to machine rounding errors. They also reported that this non-oscillatory mode were the most unstable mode at $0.594<\mathrm{g}^{*}<0.607$.

In asymmetric single vortex street mode (mode III), the locations of the maximum value of pressure fluctuations, velocity fluctuations in the wake are farther in the downstream, compared to corresponding locations in symmetric single vortex street mode (mode II). Such a downstream shift of the location of the maximum fluctuations can also be observed in the location of the downstream recirculation bubbles near the center line. In asymmetric single vortex street mode (mode III), this pair of recirculation bubbles as shown in Figure 11(d), although asymmetric about the centerline $(y=0)$, are pushed further downstream by the gap flow, compared to that in symmetric single vortex street mode (mode II) shown in Figure $11(\mathrm{c})$

\section{Irregular vortex shedding mode (mode IV)}

Mode IV denotes the irregular vortex shedding mode that describes non-periodic transient wake. Referring to Figures 6(d) and 6(e), the instantaneous vorticity may show single vortex street (Figure 6(d)), or two vortex streets at higher gap ratios in the near wake region (Figure 
6(e)). This mode, as mapped in Figure 5, can be observed to happen in the gap ratio range of $0.35 \leq \mathrm{g}^{*} \leq 1.8$. At low gap ratios, this mode is observed at relatively high Reynolds numbers. However, for $\mathrm{g}^{*}>1.2$, this irregular shedding mode may exist in the Reynolds number range between two isolated synchronized in-phase shedding mode regions (mode V). In other words, within this gap range $\left(1.2 \leq g^{*} \leq 1.8\right)$, increasing or decreasing Reynolds number may both lead to the wake pattern transition from the case here of irregular shedding mode (mode IV) to periodic synchronized in-phase shedding mode (mode V).

This irregular shedding mode features non-stationary shedding frequencies as shown in Figure $7(a)$. Because the flow is non-periodic, the frequencies identified from FFT analysis of force coefficients are dependent on how long the calculation runs. In some cases, a range of dominant frequencies may be identified. In all cases, a range of low frequencies are always presented. Both drag (Figure 8(c)) and lift (Figure 9(c)) coefficients shows irregular variations with time. But they can statistically converge if the simulation runs long enough (up to $\mathrm{O}\left(10^{3}\right) \mathrm{T}_{0}$ ). This implies that the irregular vortex shedding is temporally coherent although the shedding frequency is not stationary. The correlations of $u$ (Figure 10(i)) and $v$ (Figure 10(j)) in two points indicate the flow is non-periodic, for the time scale of our study case. The instantaneous velocity profiles (Figures $10(\mathrm{k})$ and $10(\mathrm{l})$ ) in the near wake show the existence of some non-harmonic fluctuation frequencies, which can be found in the FFT analysis of the force coefficients.

For the mean flow, both asymmetric mean flow (e.g., Figure 11(e)) and apparently symmetric mean flow were observed, depending on $\mathrm{g}^{*}$ and Re. In this mode (mode IV), the pair of recirculation bubbles in downstream near the centerline were pushed further downstream compared to that in asymmetric single vortex street mode III (Figure 11(d)). This implies that stronger (compared to that in asymmetric single vortex street mode) gap flow is necessary for the transition from asymmetric periodic shedding mode (mode III) to irregular shedding mode (mode IV). The fluctuations of pressure, $x$-component velocity and y-component velocity may show similar patterns belonging to either one of single vortex 
street mode (mode II), asymmetric single vortex street mode (mode III), or synchronized inphase shedding mode (mode V), depending on $\mathrm{g}^{*}$ and Re. For example, Figures 12(c), 13(c) and $14(\mathrm{c})$ show similar fluctuation patterns of irregular shedding mode (mode IV) as those of asymmetric single vortex street mode (mode III). The location of the maximum fluctuations is further downstream compared to that of asymmetric single vortex street mode (mode III). This is consistent with the shift of location of the pair of downstream recirculation bubbles near centerline mentioned above. The maximum values of the pressure and velocity fluctuations in the wake are smaller than those in asymmetric single vortex street mode (mode III).

\section{E. Synchronized in-phase vortex shedding (mode V)}

For gap ratio between 1.2 and 3.2, synchronized in-phase vortex shedding (mode $V$ ) is observed in the regions as shown in Figure 5. Figure 6(f) illustrates the instantaneous vorticity field in this mode. Synchronized like-sign vortices are shed from the two cylinders simultaneously. However, in the downstream, two vortex streets evolve into a binary-vortex street as observed and named by Williamson (1985) for a circular cylinder.

The shedding frequency in mode $\mathrm{V}$ is stationary and is apparently invariant to initial conditions or initial perturbations. The drag coefficients of the two cylinders have a phase difference of half shedding period as shown in Figure $8(d)$. This is similar to that in the symmetric single vortex street mode (mode II). The lift coefficients of two cylinders are synchronized with no phase difference, and therefore, the standard deviation of the overall (total) lift coefficient is twice of that of individual cylinder (Figure 9(d)). As shown in Figures $10(w)$ and $10(x)$, the instantaneous velocity field in the wake is periodic. The correlation of $u$ (Figure $10(u))$ and $v$ (Figure $10(v))$ in two points indicates the period for $u$ fluctuations is twice of that for $v$.

One example of the mean flow field of this mode is shown in Figure 11(f). A pair of recirculation bubbles behind each cylinder can be observed. This is similar to that of a single 
cylinder. However, the pair of recirculation bubbles behind each cylinder are asymmetric about the centerline (in y direction) of the corresponding cylinder. The recirculation bubbles are noticeably pushed towards freestream side. In the upstream, the stagnation point in the leading edge of the two cylinders $(x / D=-0.5)$ is biased towards the gap side. This suggests gap flow is stronger than the freestream flow. In the near wake, the gap flow is strong enough to separate the wake of two cylinders. But in downstream, the flow near the centerline $(y / D=0)$ gradually weakens and flow speed is the minimum in the cross section after $x>10 \sim 15 D$. This coincides with the formation of binary-vortex street in the downstream.

As shown in Figure 12(d), in this in-phase mode (mode $\mathrm{V}$ ), the magnitude of pressure fluctuations in the gap side is slightly weaker than that in freestream side in the near wake region. The large fluctuations occur only for a short distance in the gap side. Fluctuations of x-component velocity (Figure 13(d)) in the gap side are slightly stronger in the near wake region. Two separated rows of opposite-sign vortices in the downstream (Figure 6(f)) may weaken the main flow in $+x$ direction near the centerline because both rows of vortices facilitate the flow in $-x$ direction. Therefore, a region with low mean flow near the centerline is observed after the formation of binary-vortex street. Strong fluctuations of $y$-component velocity (Figure 14(d)) can only be observed in short distance in the downstream behind each cylinder. After the binary vortex street is formed, the fluctuation becomes weaker very quickly.

\section{F. Synchronized anti-phase vortex shedding mode (mode VI)}

In this synchronized anti-phase vortex shedding mode (mode VI), opposite-sign vortices are shed from the two cylinders simultaneously. Two parallel vortex streets are formed in the downstream as shown in Figure 6(g). Unlike the synchronized in-phase mode (mode V), the two vortex streets in anti-phase mode can sustain longer distance in the downstream. As shown in Figure 5, for $\mathrm{g}^{*} \geq 4$, anti-phase mode would be obtained for all Reynolds numbers studied when vortex shedding is observed. For $1.8<\mathrm{g}^{*}<3.5$, anti-phase mode was only 
observed at high Reynolds numbers. Lowering Reynolds number in this $\mathrm{g}^{*}$ range may lead to in-phase mode (mode V).

In this anti-phase mode (mode $\mathrm{VI}$ ), the flow is also periodic with one stationary shedding frequency. The drag forces of the two cylinders are identical once flow becomes periodic as shown in Figure 8(e). The lift forces of the two cylinders are anti-phased. This means the overall (net) lift force at any time is always zero once flow becomes periodic as shown in Figure 9(e).

The mean flow field in this mode $\mathrm{VI}$ (Figure $11(\mathrm{~g}))$ is symmetric with the centerline $(\mathrm{y}=0)$. A pair of recirculation bubbles behind each cylinder can be observed. However, this pair of recirculation bubbles are asymmetric about the centerline ( $y$ direction) of each cylinder. This is similar to that in the in-phase mode (mode V). The mean flow velocity in the centerline $(y=0)$ is considerably larger than that in the centerline (in y direction) of individual cylinder. This is different from that of the in-phase mode (mode $V$ ), where the mean velocity in the centerline is usually quite low. When a pair of opposite-sign vortices are shed from the gap side simultaneously, the fluid in the region between the pair of vortices is accelerated because both vortices tend to push the fluid in this region in the same direction $(+x)$. The elevated flow velocity in this region will lead to lower pressure, which in turn, accelerates flow towards this region. The result is higher mean flow velocity near the centerline.

Pressure fluctuations in the gap side are generally stronger and last a longer distance downstream, as compared to those in the freestream side (Figure 12(e)). For x-component velocity fluctuations (Figure 13(e)), their values are generally larger at the gap side than those in the freestream side, although in some cases, the maximum value of $\overline{u^{\prime 2}} / U_{0}^{2}$ may be located in freestream side. Unlike the synchronized in-phase mode (mode V), regions with large $\overline{u^{\prime 2}} / U_{0}^{2}$ at the gap side are isolated from the counterparts in the freestream side. This suggests that in this anti-phase mode (mode $\mathrm{VI}$ ), the interaction of the wakes of two cylinders are probably dominated by the interaction of the pair of opposite-sign gap vortices, 
while in the in-phase mode (mode V), the interaction is instead dominated by merging of likesigned vortices from gap side and freestream side. The y-component velocity fluctuations (Figure 14(e)) last a farther distance in the downstream than those in the in-phase mode (mode $\mathrm{V}$ ). But the maximum value of $\overline{v^{\prime 2}} / U_{0}^{2}$ is usually lower than that in the in-phase mode (mode $\mathrm{V}$ ) at neighboring $\operatorname{Re}$ or $\mathrm{g}^{*}$.

\section{G. Synchronized in-phase dominated vortex shedding with low frequency modulation mode (mode VII)}

For $0.7<g^{*}<1.5$ and $45<\operatorname{Re}<65$, the wake features a synchronized in-phase dominated vortex shedding with apparently low frequency modulations on it. The instantaneous vorticity visualization indicates the vortex shedding is synchronized most of the time (Figure $6(\mathrm{~h}))$. In other periods, some small phase difference in vortex shedding from two cylinders may be observed periodically.

In this mode (mode VII), FFT analysis of the lift coefficient indicates several peaks in the frequency as shown in Figure $7(b)$. The dominant low frequency, which is usually one order lower than the shedding frequency, equals or very close to the difference of the highest two peaks (which are usually contiguous). The drag coefficient in this mode (mode VII) is shown in Figure 8(f). The phase difference of drag coefficient of these two cylinders is about half period of the low frequency. The mean drag coefficient is identical for the two cylinders. While the lift coefficients (Figure 9(f)) of the two cylinders are mostly in-phase. For example, for $g^{*}=1.2$ and $R e=50$, the standard deviation of the lift coefficient of individual cylinder is about 0.533 . For the net overall lift coefficient, the standard deviation is 0.0928 . This indicates that they are not exactly in-phased all the time. The origin of the low frequency modulation is probably due to the beat frequency from the two peak shedding frequencies. These two frequencies are probably linked to two different unstable instability modes. The investigation on the origin of such low frequency modulation is ongoing. 
Low frequency modulation can be readily observed in the instantaneous flow field. As shown in Figures $10(\mathrm{~m})$ and $10(\mathrm{n})$, the correlations of $u$ and $v$ in two points indicate the existence of multiple periods in the fluctuations. The flow itself is apparently periodic as shown in Figures $10(0)$ and $10(p)$. But the period is far larger than the vortex shedding period.

The mean flow field in this mode (Figure 11(h)) is similar to that of synchronized in-phase mode (mode $\mathrm{V}$ ). The low frequency modulation apparently does not alter the characteristics of the mean flow. Pressure fluctuations in the freestream side are substantially stronger than that in the gap side as shown in Figure 12(f). However, fluctuation of the $\mathrm{x}$-component velocity shows an opposite trend; it is far stronger and it lasts farther downstream in the gap side (Figure 13(f)). The fluctuations of y-component velocity (Figure 14(f)) indicate that as it goes downstream, the two vortex streets evolve into single binary-vortex street, which is similar to the observation in the in-phase mode (mode V), without low frequency modulation.

\section{H. Synchronized anti-phase dominated vortex shedding with low frequency modulation mode (mode VIII)}

In this mode VIII, comprising of synchronized anti-phase dominated vortex shedding and low frequency modulation, the pair of gap vortices are not always shed exactly simultaneously, as depicted in Figure 6(i). Periodically, one of them may be shed slightly ahead of the other. So in the downstream, the pairs of opposite-sign gap vortices are usually not exactly aligned in y direction. Likewise, the vortices from freestream sides do not usually align in y direction either. This mode can be observed at $1.8 \leq \mathrm{g}^{*} \leq 3.5$ and $45 \leq \mathrm{Re} \leq 57.5$. Increasing Reynolds number in this mode may lead to synchronized in-phase shedding mode $V$, instead of the synchronized anti-phase mode VI.

The shedding frequency of this mode is similar to that of synchronized in-phase dominated vortex shedding with low frequency modulation mode (mode VII). The dominant low frequency, which is also usually one order lower than the shedding frequency, equals or very close to the difference of the highest two peaks. The drag coefficient in this mode (Figure 8(g)) shows clearly the existence of low frequency modulation. The overall instantaneous 
drag has much smaller fluctuations. This is similar to that in mode VII. The mean drag for each of the two cylinders is identical. The lift coefficients of the two cylinders in this mode (Figure $9(\mathrm{~g})$ ) are mostly anti-phased to each other. As a result, the fluctuations of instantaneous net lift is are smaller than those of individual cylinders.

Similar to that in mode VII, low frequency modulation is clearly observed in the instantaneous velocity field. In the instantaneous $v$ plot in near wake region as shown in Figure $10(\mathrm{t})$, the long period of $v$ fluctuations is significant.

In Figure 11(i), it shows that the mean flow field is similar to that of synchronized anti-phase mode VI because apparently the low frequency modulation does not affect the mean flow. The pressure fluctuations, in Figure 12(g), at the gap side are noticeably stronger than those in the freestream side. This is contrary to that in mode VII. However, similar to mode VII, the fluctuations of x-component velocity (Figure $13(\mathrm{~g})$ ) in the gap side are stronger than those in the freestream side. Compared to the spatial distribution of $\overline{u^{\prime 2}} / U_{0}^{2}$ in anti-phase mode $\mathrm{VI}$, as observed in Figure 13(e), the regions with large fluctuations in the gap side and the freestream side are found to merge with each other. Spatial distribution of $\overline{v^{\prime 2}} / U_{0}^{2}$ (Figure 14(g)) has a similar pattern as that in anti-phase mode without low frequency modulation (Figure 14(e)). The major difference is that the two regions with large fluctuations in the wake are connected, and not isolated, when low frequency modulation presents itself in this mode. This suggests strong interaction between pair of vortices shed from freestream side and therefore the gap vortices are not well developed in the downstream in this mode.

\section{Asymmetric synchronized anti-phase vortex shedding mode (mode IX)}

In the earlier-discussed synchronized anti-phase vortex shedding mode VI, two vortex streets are symmetric about the gap centerline $(y / D=0)$. But at a Reynolds number just slightly higher than the critical Reynolds number within $2.2 \leq \mathrm{g}^{*} \leq 3.5$, two asymmetric vortex streets were observed. The vortex street is biased towards one of the cylinders in $y$ direction. Increasing Reynolds number will lead to the synchronized anti-phase shedding 
with low frequency modulation mode VIII. For example, as shown in Figure 6(j), the two vortex streets are shifted towards cylinder 1 . Of course, similar to that in asymmetric single vortex street mode III, the direction can be found to bias either to cylinder 1 or to cylinder 2 . But the direction of the deflection will not flip; in other words, if the wake is biased to cylinder 1 , it will be biased to cylinder 1 without switching back.

The two cylinders have an identical shedding frequency as in the symmetric anti-phase mode VI. But the drag coefficient is different for the two cylinders due the asymmetricity of the wake. For example, with $\mathrm{g}^{*}=2.8$ and $\mathrm{Re}=45$ (Figure $8(\mathrm{~h})$ ), cylinder 1 has a mean drag coefficient of 1.968 (standard deviation is 0.0022 ) and 2.003 (standard deviation is 0.0035 ) for cylinder 2. The lift coefficients (Figure 9(h)) of the 2 cylinders are anti-phased to each other, but does not cancel out. Hence, the net lift coefficient is not zero, which is similar to the asymmetric single vortex street mode III.

The mean flow (Figure 11(j)) indicates that the recirculation bubbles behind cylinder 1 is noticeably larger than those behind cylinder 2 when the wake behind cylinder 1 is narrower. The asymmetric spatial distributions of fluctuations of pressure, $x$-component velocity and $y$ component velocity are clearly shown in Figures $12(\mathrm{~h}), 13(\mathrm{~h})$ and $14(\mathrm{~h})$ respectively. In this particular case, fluctuations behind cylinder 2 are stronger than those behind cylinder 1 in general.

\section{The role of gap flow in wake pattern transitions}

As discussed in Section IV, both gap ratio and Reynolds number play an important role in determining the wake pattern and vortex shedding modes. In some situations, these two factors are correlated. For example, at $\mathrm{g}^{*}=0.4$ and $\mathrm{Re}=60$, increasing $\operatorname{Re}$ or $\mathrm{g}^{\star}$ may give similar wake pattern transition path: from symmetric single vortex street to asymmetric single vortex street flow, then to irregular flow. This implies, under certain conditions, it may be possible to express the combined effects of changing Re and changing $\mathrm{g}^{*}$, in some reduced or aggregated physical parameters to help explain the feature. In this study, we found the 
gap flow to give good indications for wake pattern transitions among these 9 different modes.

One overall parameter for gauging gap flow is the length-weighted averaged mean flow ( $\mathrm{x}$ component) in the whole gap from $(0,-0.5 \mathrm{~g})$ to $(0,0.5 \mathrm{~g})$, which is defined as:

$U_{\text {gap }}=\frac{1}{g} \int_{-0.5 g}^{0.5 g} \overline{U_{x}} d y$

Figure 16 plots the averaged mean gap flow at $R e=50,75,100,150$ and 200 . Lengthweighted averaged $\overline{U_{x}}$ in the whole cross section at $\mathrm{x}=0$ from $(0,-15 \mathrm{D})$ to $(0,15 \mathrm{D}), 1.071 \mathrm{U}_{0}$ (based on the blockage ratio of 0.0667 ), is marked as the dashed line in the plot. As shown in the figure, the mean gap flow, $U_{\text {gap }} / U_{0}$, increases with $\mathrm{Re}$ at all gap ratios. It also increases with gap ratio up to a maximum value, then decreases. The wake is found to transitioned from synchronized in-phase mode (mode V) to eventually the synchronized antiphase mode (mode $\mathrm{VI}$ ) at the maxima (marked as circles in Figure 16) and then maintains this anti-phase mode thereafter in the gap ratio range studied. In other words, at any given Reynolds number, increasing the gap ratio will lead to an increase in mean gap flow initially. When the gap flow reaches the maximum, the flow regime will switch eventually to the synchronized anti-phase mode (mode VI). Similarly, at a given gap ratio, increasing Re will lead to increase of the mean gap flow, increasing the $U_{\text {gap }}$ value, such that when the $U_{\text {gap }} / U_{\text {o }}$ crosses over a specific curve joining the points of maxima (marked by circle in Figure 16), the vortex shedding is always in the synchronized anti-phase mode VI.

In the regime of low gap ratios less than $0.7\left(\mathrm{~g}^{*}<0.7\right)$, the gap flow is weak and probably not strong enough to trigger vortex shedding from the gap side. As shown in Figure 16, the mean gap flow is always lower than the length-weighted averaged $\overline{U_{x}}$ in the whole cross section at $x=0,1.071 U_{0}$, for this regime, $g^{*}<0.7$. The wake is therefore dominated by vortex shedding from the shear layers in the freestream side and a single vortex street was observed at low gap ratios. 
In addition to mean gap flow, the mean velocity profile in the gap also varies with gap ratio and Reynolds number. Figure 17 shows different profiles of mean x-component velocity in the middle $(x=0)$ of the gap. At low gap ratios (for example, at $g^{*}=0.4$ as shown in Figure $17(a))$, velocity profile features a maximum value at the gap center $(y=0)$. The profile is analogous to that of steady-state flow through a flat channel. When increasing the gap ratio, a jet-like velocity profile near the cylinder surface of each cylinder gradually develops. Increasing Reynolds number may also help the development of this velocity profile when $g^{*}>0.7$. For example, as shown in Figures $17(\mathrm{~b})$ and $17(\mathrm{c})$, at $\mathrm{g}^{*}=1$ and 2.2 , the near-wall velocity profiles are gradually developed with the increase of Reynolds number. At relatively large gap ratios, for example at $\mathrm{g}^{*}=5$ (Figure $17(\mathrm{~d})$ ), such a near-wall velocity profile is fully developed and the value of $\overline{U_{x}} / U_{0}$ in the center of the gap $(\mathrm{y}=0)$ appears invariant to Reynolds number. At the regime of low gap ratios where $\mathrm{g}^{*}<0.7$, such a near-wall velocity profile does not exist and gap flow is similar to laminar flow in a flat channel, so the wake is dominated by the shear layers in the freestream side. Increasing Reynolds number or increasing gap ratio within this regime will only elevate the peak velocity in the center as indicated in Figure 17(a). This means, if the peak velocity is strong enough, unstable modes may be developed from gap flow in the downstream. Because of the single peak in the profile, the flow corresponding to the unstable modes may tend to switch to one side in the downstream causing a pitchfork bifurcation. This means very likely, the unstable modes developed from gap flow is non-oscillatory in this case. The asymmetric single vortex street mode may therefore be triggered if the non-oscillatory unstable mode is not strong compared to the oscillating modes corresponding to the shear layers in the freestream side. But if $\operatorname{Re}$ is even higher, or the gap is even larger, the instability due to the gap flow may be in the same order of that of the shear layers in the freestream side, the non-oscillatory unstable modes may therefore destroy or alter the periodic flow in the wake and the flow regime may be transitioned to irregular mode. As shown in Figure 5 , up to $R e=125$, irregular mode is always transitioned through asymmetric single vortex mode when increasing $\mathrm{Re}$ or $\mathrm{g}^{*}$ for this regime where $\mathrm{g}^{*}<0.7$. For $\operatorname{Re}>125$, possibly due to other possible unstable modes, or the resolution 
limitations of $\mathrm{g}^{*}$ and $\mathrm{Re}$ in this study, or $3 \mathrm{D}$ transitions, the flow is transitioned to irregular mode without going through asymmetric mode.

As discussed earlier, however, in the other regime where gap ratio is larger than 0.7 , gap vortices could be shed from each cylinder, forming possibly two vortex streets in the near wake. As shown in Figure $17(\mathrm{~b})$, at $\mathrm{g}^{*}=1$, the velocity profile in the center is flat, and the velocity profile near the cylinder wall in the gap side is still developing due to insufficient gap spacing. In this situation, the periodic vortex shedding from the gap side may be destroyed by the unstable modes developed from the flat profile in the gap center because the velocity near the wall in gap side and that in the center of the gap are in the same order. Hence, increasing Reynolds number or increasing the gap ratio, which essentially increases the gap flow, in this regime would lead to irregular shedding mode too. However, further increasing the gap ratio, the near wall velocity profile can be so well developed, as shown in Figure $17(c)$, such that the velocity near the wall is higher than that in the gap center. This means the periodic vortex shedding from the gap side may unlikely be destroyed by the unstable modes that arose from the gap flow profile near the gap center. Therefore, irregular shedding may only exists in a limited regime within these conditions explained. As shown in Figure 5, for the regime of gap ratio larger than 2, irregular shedding mode does not exist in the Reynolds number/range studied. In the range of $1.2<g^{*}<2$, further increasing $g^{*}$ or $\operatorname{Re}$ may lead to wake pattern transition from irregular shedding mode to periodic vortex shedding, due to the development of the near-wall velocity profile in the gap side. Comparing Figures $17(\mathrm{c})$ and $17(\mathrm{~d})$, it can be found that the near-wall velocity profile in Figure $17(\mathrm{c})$ is still not fully developed for $\operatorname{Re} \leq 100$. When the near wall velocity profile is fully developed as shown in Figure $17(\mathrm{~d})$, the mean gap flow will decrease with increasing gap ratio. This indicates the interaction of the two cylinders becomes weaker with increasing gap ratio then.

Although the mean gap flow and mean velocity profile in the gap could explain physically quite well the transitions across various modes, we are not able to find good evidences or 

modes yet. Investigations is still on going.

\section{The impact of gap ratio and Reynolds number on shedding frequency and force coefficients}

\section{A. Impact on shedding frequency}

Figure 18 plots the shedding frequency (Strouhal number) against various gap ratios and Reynolds numbers. Data points for irregular shedding mode (mode IV) and modes with low frequency modulations (mode VII, VIII) were not included. It is interesting to note that the Strouhal number is clustered into two separate distinct groups. For gap ratios $g^{*} \leq 0.7$, all shedding frequencies are found below the line corresponding to $g^{*}=0$ ( $D$ was used as characteristic length for all cases, for consistent comparison). Conversely, for $g^{*}>1$, all the shedding frequencies are found above the line for that of the single cylinder. For $\mathrm{g}^{*}=0.8$ and 1 , the shedding frequencies are between these two clusters.

For the regime of $g^{*} \leq 0.7$, shedding frequency generally decreases with gap ratio and increases with Reynolds number. As discussed earlier, in the single vortex street mode II or III (symmetric or asymmetric), only one vortex street is observed in the downstream. The gap flow usually is not strong. As there is no periodic vortex shedding from the gap side, the gap flow is acting as a damping system to the periodic flow from the vortex shedding at the freestream side of the cylinder. This is evidenced in the spatial distribution of velocity fluctuations. For example, as shown in Figures 14(a) and 14(b), the regions with large $\overline{v^{\prime 2}} / U_{0}^{2}$ are pushed downwards by the gap flow when wake mode changes from the symmetric single vortex street mode II to the asymmetric mode III. Such a damping mechanism will effectively reduce the shedding frequency of the freestream side. Increasing gap ratio at a given Reynolds number, will increase the gap flow and enhance the damping. Therefore, at a given Reynolds number, the shedding frequency decreases with gap ratio. When increasing Reynolds number at a given gap ratio, the mean gap flow also increases. This would increase the damping too. However, increasing Reynolds number could also 
cause the shear rate in the freestream side to increase and thereby, causing the shedding frequency to increases. It seems under the simulation conditions of this study, the increase in shedding frequency due to Reynolds number increase is more significant than the damping from the gap flow. With a blockage of $1 / 15$ in this study, the length-weighted averaged mean $x$-component velocity in the whole section at $x=0$ is about $1.071 U_{0}$. As shown in Figure 16, for $g^{*} \leq 0.7$ the mean gap flow is all lower than $1.071 \cup_{0}$. This implies the gap flow is not as strong as the flow in freestream side yet. Therefore, the increase in damping effects of the gap flow may be cancelled by the increasing in shear of the flow in freestream side. So, the shedding frequency increases with Reynolds number in this gap ratio range. Note that for $\mathrm{g}^{*}=0$ and 0.1 , Strouhal number slightly decreases with $\mathrm{Re}$ for $\operatorname{Re}>110$. This is probably due to $2 \mathrm{D}$ to $3 \mathrm{D}$ transition, which occurs at lower Reynolds number when $\mathrm{g}^{*}$ is very small.

For the regime of $g^{*}>1$, the shedding frequency is higher than that of a single cylinder at same Reynolds number. As shown in Figure 16 , for $\mathrm{g}^{*}>1.2$, the mean gap flow velocity is higher than the average in the whole section $\left(1.071 \mathrm{U}_{0}\right)$. This means the gap flow is actually stronger than the flow in the freestream side for $g^{*}>1.2$, and this is supported clearly by the comparison plots of the near-wall mean velocity profiles between the gap side and the freestream side for 2 cases of $g^{*}=2.2$ and $g^{*}=5$ in Figure 19. It can be found that gap side has higher peak values with thinner profiles than the freestream side. This means higher shear rate in the gap side than the shear rate in the freestream side, which is similar to that for single cylinder. The stronger gap flow will increase the shedding frequency of gap vortices, leading subsequently to an increase of the shedding frequency of the observed synchronized vortex shedding. Putting these reasons together, the shedding frequency for this regime of $\mathrm{g}^{*}>1.2$ would therefore be higher than that for a single cylinder. Shedding frequency decreases with gap ratio for both the synchronized in-phase mode $V$ and the antiphase modes VI. This is very likely due to the transitions of the near-wall velocity profiles. For example, as shown in Figure 19 , at $\mathrm{g}^{*}=2.2$, the near-wall mean velocity in the gap side is 
substantially stronger than that in the freestream side. However, at $g^{*}=5$, the difference becomes smaller although it is still stronger in the gap side. When the gap ratio increases further, the interaction of the two cylinders becomes weaker and the shedding frequency will then be comparable to that of a single cylinder. It is further noted that at a given Reynolds number, if increasing gap ratio leads to wake pattern transition from in-phase mode to antiphase mode, the Strouhal number may be found to increase. For example, at $\operatorname{Re}=100$ and $\mathrm{g}^{*}=2$, the synchronized in-phase mode $\mathrm{V}$ is observed. Increasing $\mathrm{g}^{*}$ to 2.5 will lead to wake pattern transition to the synchronized anti-phase mode VI. The Strouhal number will then increase, instead of decreasing, as found in Figure 18. Apparently, the anti-phase mode VI tends to have a slightly, but consistently, higher shedding frequency than the in-phase mode V.

\section{B. Impact on mean drag coefficient}

The impact of $\operatorname{Re}$ and $\mathrm{g}^{*}$ on mean drag coefficient is shown in Figure 20. In most cases, even in the irregular shedding mode IV, the mean drag coefficient for the two cylinders are almost identical, except for the asymmetric single vortex street mode III and the asymmetric synchronized antiphase dominated mode IX. In the plots in Figure 20, data points from all the modes are included. For asymmetric modes (mode III and mode IX), the average of the mean drag coefficient from two cylinders is used.

For the regime of $g^{*} \leq 0.7$, at low Reynolds numbers $(\leq 40)$, the mean drag coefficient increases with gap ratio. For $\operatorname{Re}$ up to 60 , drag coefficient is larger than that of $g^{*}=0$. For $\mathrm{g}^{*}=0.1$, the drag coefficient is consistently larger than that of $\mathrm{g}^{*}=0$ at all Reynolds numbers. At low Reynolds number and low gap ratio, the gap flow is weak and confined by the single vortex street originated from the freestream side. As shown in the mean velocity plot (Figure 11(c)), gap flow pushes the pair of recirculation bubbles downstream near the centerline without breaking them. This means the gap flow essentially increases the effective blocking area and therefore may increase the drag. For $\mathrm{g}^{*}=0.35$, as shown in Figure 20 , there is a sharp decrease of drag coefficient at about $\operatorname{Re}=80 \sim 90$. This corresponds to the wake mode 
transition from the symmetric single vortex street mode II to the asymmetric mode III. The drag then increases sharply at $\operatorname{Re}=130 \sim 140$. This corresponds to the wake mode transitioning back to the symmetric single vortex street mode II. For other asymmetric cases, the variations in drag coefficient is not so significant.

For the regime of $g^{*}>1$, drag coefficient decreases with gap ratios. This is probably due to the gap flow interaction of the two cylinders. Before the near-wall velocity profile is fully developed in the gap, the gap flow is considerably stronger than that in the freestream side as indicated in Figure 19(a). Such an imbalance may elevate the drag. When the two cylinders are separated with larger gap ratios, the interaction then becomes weaker, and the differences in the mean velocity profiles in the gap side and the freestream side become less significant. Therefore, eventually the drag coefficient will tend towards that of a single cylinder.

\section{Impact on mean lift coefficient}

The mean lift coefficient of cylinder 1 , which represents the force exerted in cylinder 1 in $y$ direction, is shown in Figure 21. Except for the two asymmetric modes and irregular mode (modes III, IX and IV), the mean lift coefficient of cylinder 2 has the identical magnitude but opposite sign as compared to that of cylinder 1 , and thereby resulting in the mean net (overall) lift force to be 0 . Overall, the mean lift force decreases with the gap ratio, and Reynolds number. For the regime of $\mathrm{g}^{*} \leq 0.7$, when increasing gap ratio or increasing Reynolds number, the gap flow is generally stronger and therefore damping effects of gap flow is more pronounced. This may effectively decrease the mean lift. For the other regime of $g^{*}>1$, the interaction between the two cylinders generally becomes less significant when increasing $\mathrm{g}^{*}$ or $\mathrm{Re}$, and hence, lower mean lift forces will be observed. For most cases, the two cylinders are repulsive (mean lift force for cylinder 1 is positive, and negative for cylinder 2). But for $g^{*}>2.5$ and $\operatorname{Re}>150$, the two cylinders can be weakly attractive, instead of repulsive. This may be linked to the low pressure regions between the paired gap vortices in anti-phase mode VI. The exact causes for this is still not well understood yet. 


\section{Conclusions}

The wake patterns of 2 side-by-side square cylinders, under the conditions of gap ratio $0 \sim 10$ and Reynolds number 16 200, were systematically studied to advance the knowledge of the interactions of two slant blunt bodies at low Reynolds numbers. From the studies, 9 different possible wake patterns and vortex shedding modes were identified based on various criteria including visualization of vortex shedding, existence of stationary shedding frequency, instantaneous and mean drag coefficient, instantaneous and mean lift coefficient, instantaneous flow field, mean flow field, spatial distribution and patterns of pressure fluctuations $\left(\overline{p^{\prime 2}} /\left(\rho^{2} U_{0}^{4}\right)\right)$, x-component velocity fluctuations $\left(\overline{u^{12}} / U_{0}^{2}\right)$, and y-component velocity fluctuations $\left(\overline{v^{\prime 2}} / U_{0}^{2}\right)$. A map on the envelopes of $\operatorname{Re}$ and $\mathrm{g}^{*}$ for individual modes and their transitions was derived.

Compared to the wake of 2 side-by-side circular cylinders at low Reynolds numbers (Kang, 2003), 3 additional modes, the in-phase dominated shedding mode with low frequency modulation (mode VII), the anti-phase dominated shedding mode with low frequency modulation (mode VIII), and the asymmetric anti-phase mode (mode IX), were observed in this study. The corresponding Re-g* ranges for other 6 modes were noticeably different. But the transition path from one mode to another among these 6 modes were similar when changing $\operatorname{Re}$ and/or $g^{*}$. The 3 new modes added additional transition paths for these 6 modes for square cylinders.

Steady-state wake features steady-state flow field without vortex shedding and can be observed at relatively very low Reynolds numbers. All other 8 modes are transient with vortex shedding. Irregular shedding mode features non-stationary shedding frequencies. It can have either single irregular vortex street $\left(0.35 \leq g^{*} \leq 0.7\right)$ or two vortex streets $\left(0.7<g^{*} \leq 1.8\right)$. Symmetric and asymmetric single vortex street modes are observed at $g^{*}<1$. Both modes feature a single vortex street and the flow is periodic. The difference is in the symmetricity of the wake. In asymmetric single vortex street mode, the wake is biased towards one cylinder along with the formations of a wide wake and a narrow wake behind 
two cylinders. The mean flow is resultantly biased towards one cylinder. The asymmetricity in asymmetric single vortex street mode is probably due to the unstable non-oscillatory models developed from the channel flow like velocity profile of the gap flow. For $g^{*}>0.7$, two vortex streets were also observed. For gap ratio larger than 1, only two vortex streets were observed. Periodic vortex shedding from two cylinders can be synchronized in an in-phase manner or in an anti-phase manner. Low frequency modulations for both in-phase and antiphase modes can be found in a very limited gap ratio and Reynolds number range. The origin of the low frequency modulation is probably the interference (beat frequency) of the two peak shedding frequencies. Asymmetric synchronized anti-phase mode exits at $2.2 \leq g^{*} \leq 3.5$ and Reynolds number close to 45 . In this mode, the two vortex streets are biased towards one cylinder.

We found mean gap flow and mean flow profile in the gap are the key parameters to explain and understand the transitions among different modes. For $\mathrm{g}^{*} \leq 0.7$, the gap flow functions as a damper for the vortex shedding from the freestream side. When the mean gap flow is not strong, the wake will be in symmetric single vortex street mode. When mean gap flow increases, non-oscillatory unstable modes may be developed from the gap flow due to single peak velocity in the gap center and therefore the flow is deflected to one side of either cylinder, and the wake is transitioned to asymmetric single vortex street mode. Further increasing the gap flow may destroy the periodicity in the wake and the wake then transitions to irregular mode. For $\mathrm{g}^{*}>0.7$, a near-wall velocity profile near the wall of each cylinder in the gap side will gradually develop with increase of $\mathrm{g}^{*}$ and Re. The location of maximum mean velocity is shifted from the gap center towards the near-wall regions gradually. Reriodic vortex shedding from the gap side then results in in-phase or anti-phase mode. Anti-phase mode is observed when and after the mean gap flow reaches the maximum.

The impact of the gap ratio and Reynolds number on shedding frequency, drag coefficient, and lift coefficient were also studied. For $\mathrm{g}^{*} \leq 0.7$, as the gap flow mainly functions as a damper for the periodic flow, the shedding frequency decreases with gap ratio. The drag 
coefficient increase with gap ratio for Reynolds number up to 40 because the gap flow is effectively confined by the periodic flow in the wake and therefore the gap flow essentially increases the blocking area. For $\mathrm{g}^{*}>1$, shedding frequency is higher than that of single cylinder due to stronger gap flow than the flow in the freestream side. Increasing the gap ratio also generally decreases the shedding frequency due to the decreased differences in near-wall velocity profile in the gap side and in the freestream side. When gap ratio reaches 10 , it is very close to that of the single cylinder. Similar trend was found for drag coefficient for $g^{*}>1$. The mean lift force on each cylinder decreases with the gap ratio and Reynolds number. The two cylinders are repulsive except for $\mathrm{g}^{*}>2.5$ and $\mathrm{Re}>150$ under the conditions of this study.

\section{Acknowledgment}

The research was conducted in the IHPC-SMMI Joint Laboratory, comprised of the Institute for High Performance Computing (IHPC), the Southampton Marine and Maritime Institute (SMMI), and the National University of Singapore (NUS). The Joint Laboratory is funded under the Agency for Science, Technology \& Research (A*STAR) Science \& Engineering Research Council (SERC) Singapore. This work was also supported by the A*STAR Computational Resource Centre and National Supercomputing Centre (NSCC) Singapore through the use of their high performance computing facilities.

\section{References}

Agrawal, A., Djenidi, L. and Antonia, R. A., "Investigation of flow around a pair of side-by-side square cylinders using the lattice Boltzmann method," Computers \& Fluids 35, 1093-1107 (2006).

Alam, M. M., Zhou, Y. and Wang, X. W., "The wake of two side-by-side square cylinders," J. Fluid Mech. 669, 432-471 (2011).

Alam, M. M. and Zhou, Y., "Intrinsic features of flow around two side-by-side square cylinders," Physics of Fluids 25, 085106 (2013).

Barkley, D. and Henderson, R. D., "Three-dimensional Floquet stability analysis of the wake of a circular cylinder,” J. Fluid Mech. 322, 215-241 (1996). 
Bearman, P. W. and Wadcock, A. J., "The interaction between a pair of circular cylinders normal to a stream," J. Fluid Mech. 61, 499-511 (1973).

Burattini, P. and Agrawal, A., "Wake interaction between two side-by-side square cylinders in channel flow," Computers \& Fluids 77, 134-142 (2013).

Cantwell, C. D. and Barkley, D., "Computational study of subcritical response in flow past a circular cylinder," Phys. Rev. E 82, 026315 (2010)

Cantwell, C. D., Moxey, D., Comerford, A., Bolis, A., Rocco, G. Mengaldo, G., Grazia, D. D., Yakovlev, S., Lombard, J.-E., Ekelschot, D., Jordi, B., Xu, H., Mohamied, Y., Eskilsson, C., Nelson, B., Vos, P., Biotto, C., Kirby, R. M. and Sherwin, S. J., "Nektart+t: An open-source spectral/hp element framework," Computer Physics Communications 192, 205-219 (2015).

Carini, M., Giannetti, F. and Auteri, F., "On the origin of the flip-flop instability of two side-by-side cylinder wakes," J. Fluid Mech. 742, 552-576 (2014).

Choi, C. B. and Yang, K. S., "Three-dimensional instability in the flow past two side-by-side square cylinders," Physics of Fluids 25, 074107 (2013).

Franke, R., Rodi, W. and Schonung, B., "Numerical calculation of laminar vortex shedding flow past cylinders," Journal of Wind Engineering and Industrial Aerodynamics 35, 237-257 (1990).

Gerrard, J. H., "The mechanics of the formation region of vortices behind bluff bodies," J. Fluid Mech. 25, 401-413 (1966).

Jordi, B. E., Cotter, C. J. and Sherwin, S. J., "An adaptive selective frequency damping method," Physics of Fluids 27, 094104 (2015)

Kang, S., "Characteristics of flow over two circular cylinders in a side-by-side arrangement at low Reynolds numbers," Physics of Fluids 15, 2486-2498 (2003).

King, R., "A review of vortex shedding research and its application," Ocean Engineering 4, 141-171 (1977).

Kolár, V., Lyn, D. A. and Rodi, W., "Ensemble-averaged measurements in the turbulent near wake of two side-by-side square cylinders," J. Fluid Mech. 346, 201-237 (1997).

Luo, S. C., Tong, X. H. and Khoo, B. C. "Transition phenomena in the wake of a square cylinder," Journal of Fluids and Structures 23, 227-248 (2007).

Mizushima, J. and Ino, Y., "Stability of flows past a pair of circular cylinders in a side-by-side arrangement," J. Fluid Mech. 505, 491-507 (2008) 
Moukalled, F., Mangani, L. and Darwish, M., The Finite Volume Method in Computational Fluid Dynamics: An Advanced Introduction with OpenFOAM ${ }^{\circledR}$ and Matlab ${ }^{\circledR}$ (Springer International Publishing Switzerland, Switzerland, 2016) p. 621

Noack, B. R. and Eckelmann, H., "A global stability analysis of the steady and periodic cylinder wake," J. Fluid Mech. 270, 297-330 (1994).

Norberg, C., "Flow around rectangular cylinders: pressure forces and wake frequencies," Journal of Wind Engineering and Industrial Aerodynamics 49, 187-196 (1993).

Okajima, A., "Strouhal numbers of rectangular cylinders," J. Fluid Mech. 123, 379-398 (1982).

Okajima, A., in Proc. 6th Int. Conf. Flow-Induced Vibration, London, UK, 10-12 April, 1995, edited by P. W. Bearman (Balkema, Rotterdam,1995), pp. 1-7

Robichaux, J., Balachandara, S. and Vanka, S. P., "Three-dimensional Floquet instability of the wake of square cylinder," Physics of Fluids 11, 560-578 (1999).

Roshko, A., "Experiments on the flow past a circular cylinder at very high Reynolds number," J. Fluid Mech. 10, 345-356 (1961).

Sohankar, A., Davidson, L. and Norberg, C., "Numerical simulation of unsteady flow around a square two-dimensional cylinder", in Twelfth Australian Fluid Mechanics Conference, Sydney, Australia, pp. 517-520 (1995).

Sohankar, A., Norberg, C. and Davidson, L., "Numerical simulation of unsteady low-Reynolds number flow around rectangular cylinders at incidence," Journal of Wind Engineering and Industrial Aerodynamics 69-71, 189-201 (1997).

Sohankar, A., Norberg, C. and Davidson, L., "Simulation of three-dimensional flow around a square cylinder at moderate Reynolds numbers," Physics of Fluids 11, 288-306 (1999).

Sumer, B. M. and Fredsøe, J., Hydrodynamics Around Cylindrical Structures (revised edition), Advanced Series on Ocean Engineering, Volume 26 (World Scientific Publishing, Singapore, 2006)

Sumner, D., Wong, S. S. T., Price, S. J. and Paigdoussis, M. P., "Fluid behaviour of side-by-side circular cylinders in steady cross-flow," Journal of Fluids and Structures 13, 309-338 (1999).

Wang, Z. J. and Zhou, Y., "Vortex interaction in a two side-by-side cylinder near-wake," International Journal of Heat and Fluid Flow 26, 362-377 (2005).

Weller, H. G., Tabor, G., Jasak, H. and Fureby, C. "A tensorial approach to computational continuum mechanics using object-oriented techniques," Computers in Physics 12, 620-631 (1998). 

(1985).

Williamson, C.H.K., "Vortex dynamics in the cylinder wake," Annu. Rev. Fluid. Mech. 28, 477-539 (1996).

Yen, S. C. and Liu, J. H., "Wake flow behind two side-by-side square cylinders," International Journal of Heat and Fluid Flow 32, 41-51 (2011). 


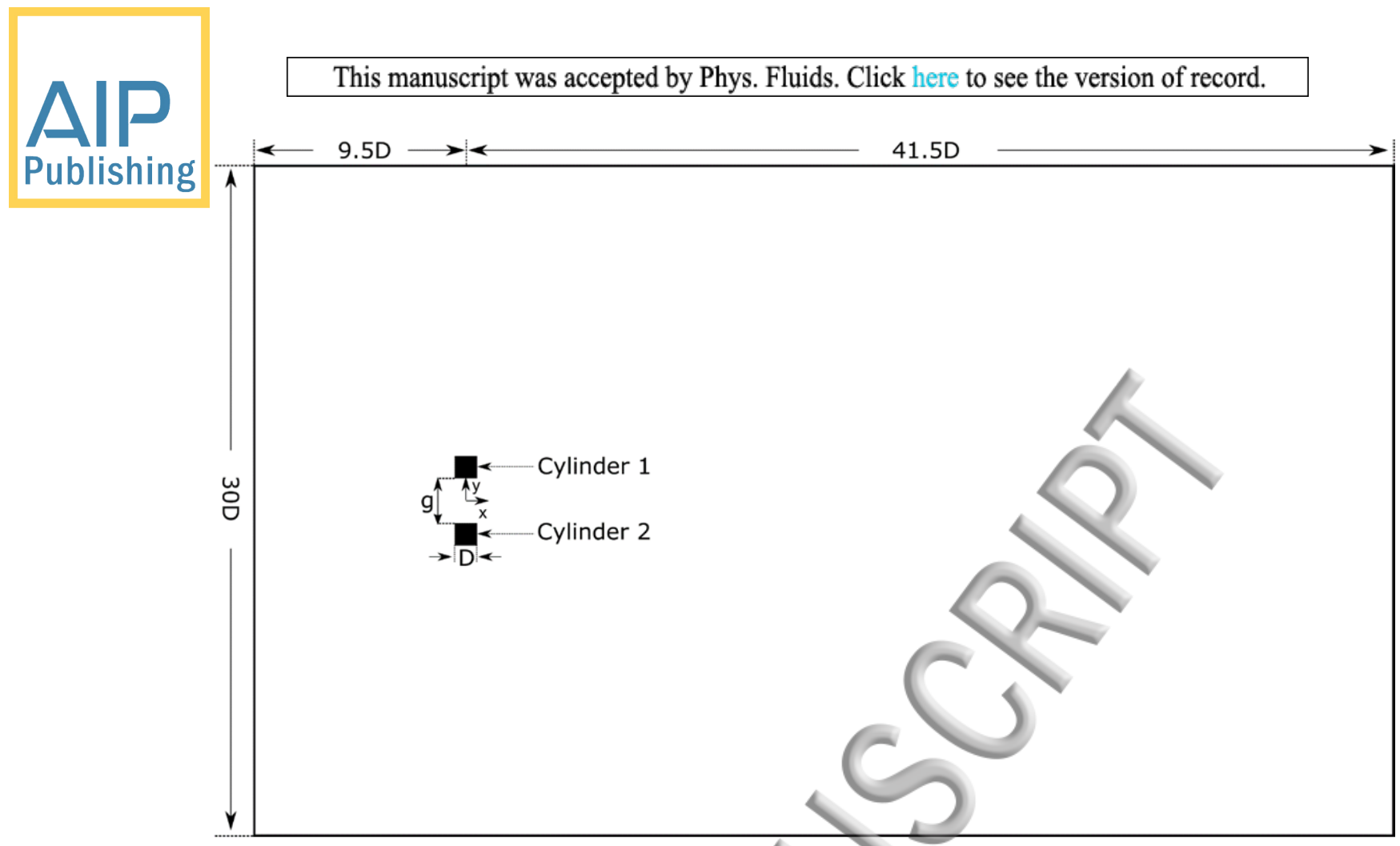

Figure 1. Illustration of problem setup and computing domain.

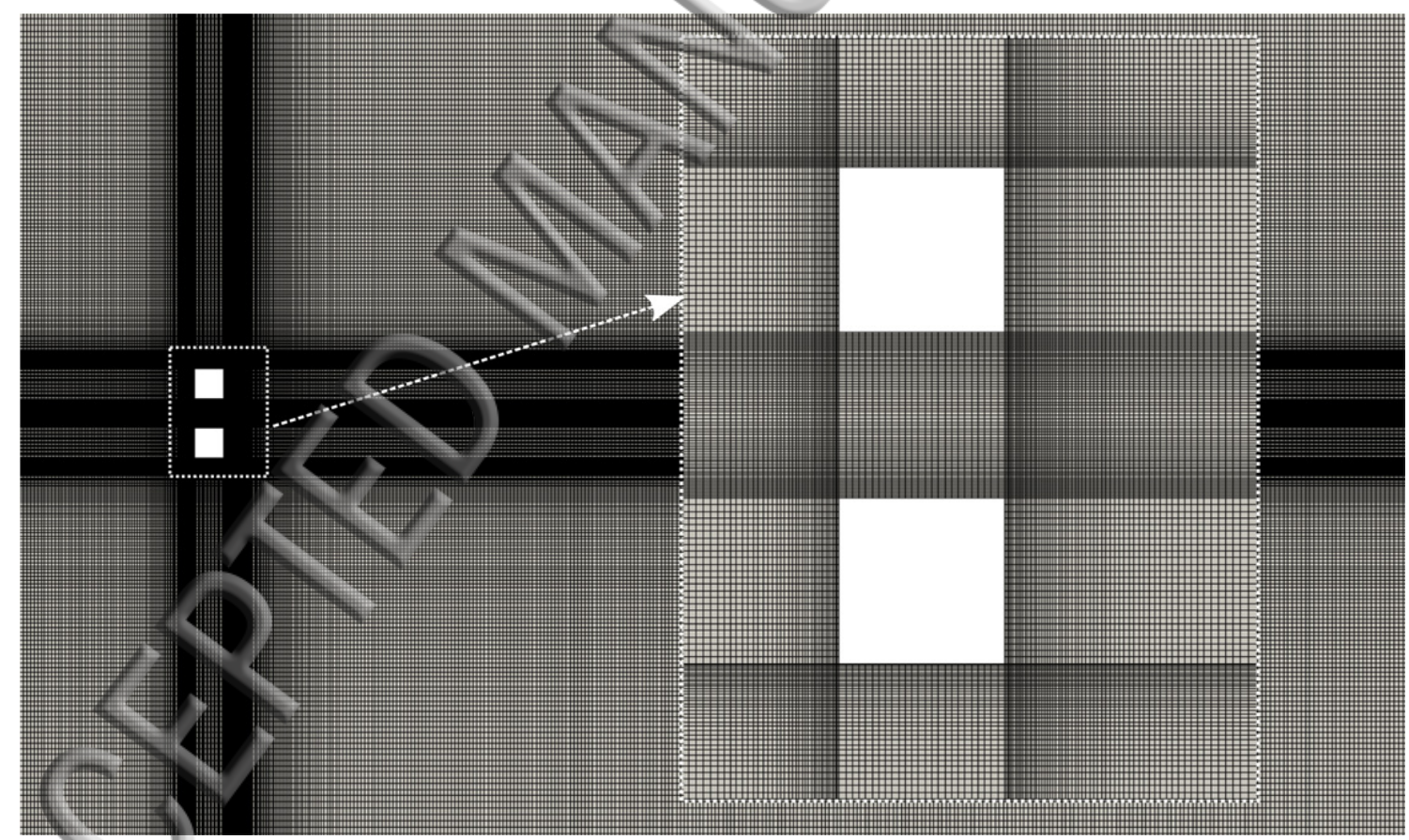

Figure 2. Illustration of mesh for $g^{*}=1$ with zoom-in view near the cylinder 


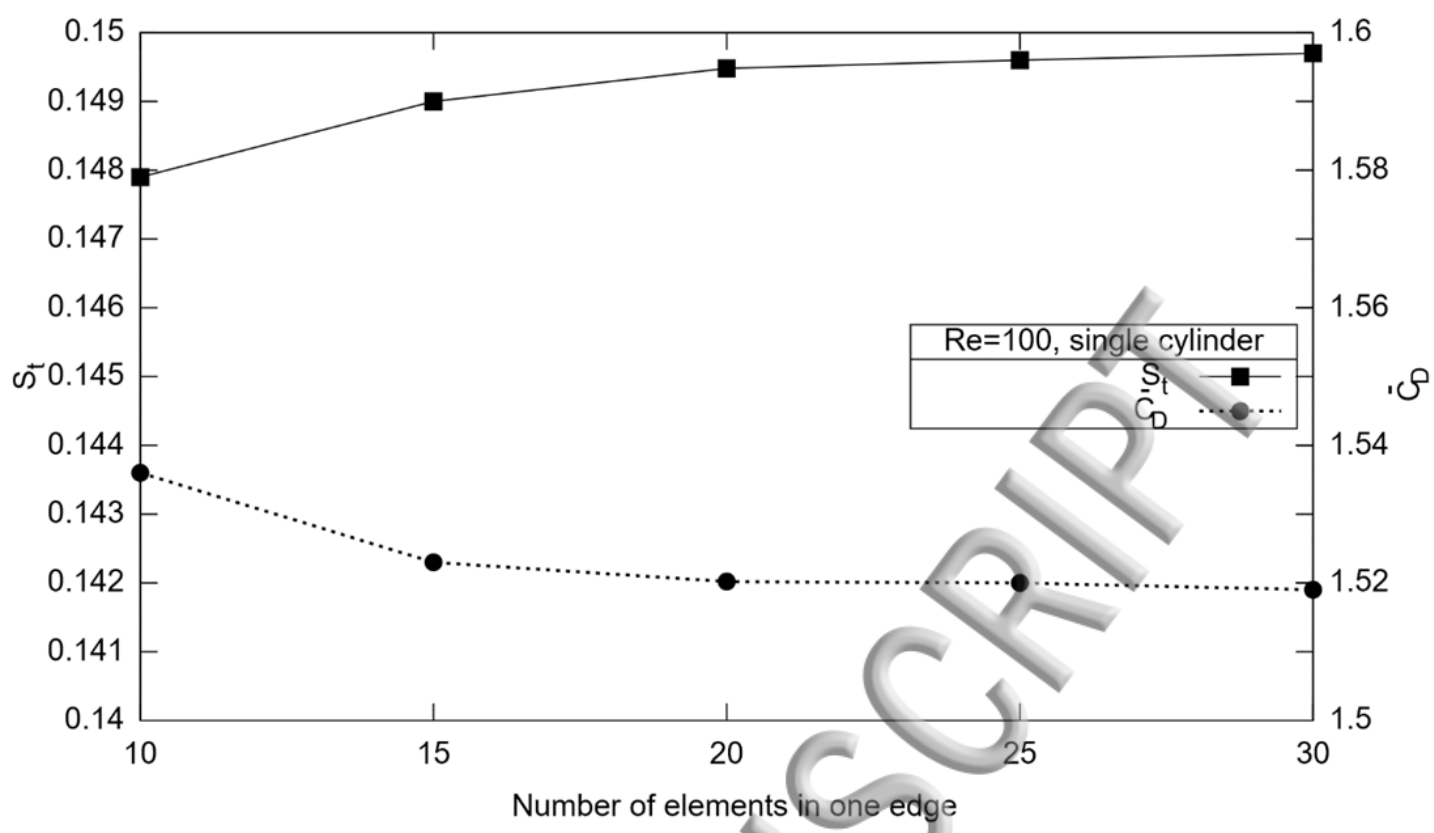

Figure 3. Convergence check for mesh size on cylinder surface (single cylinder, $R e=100$ )
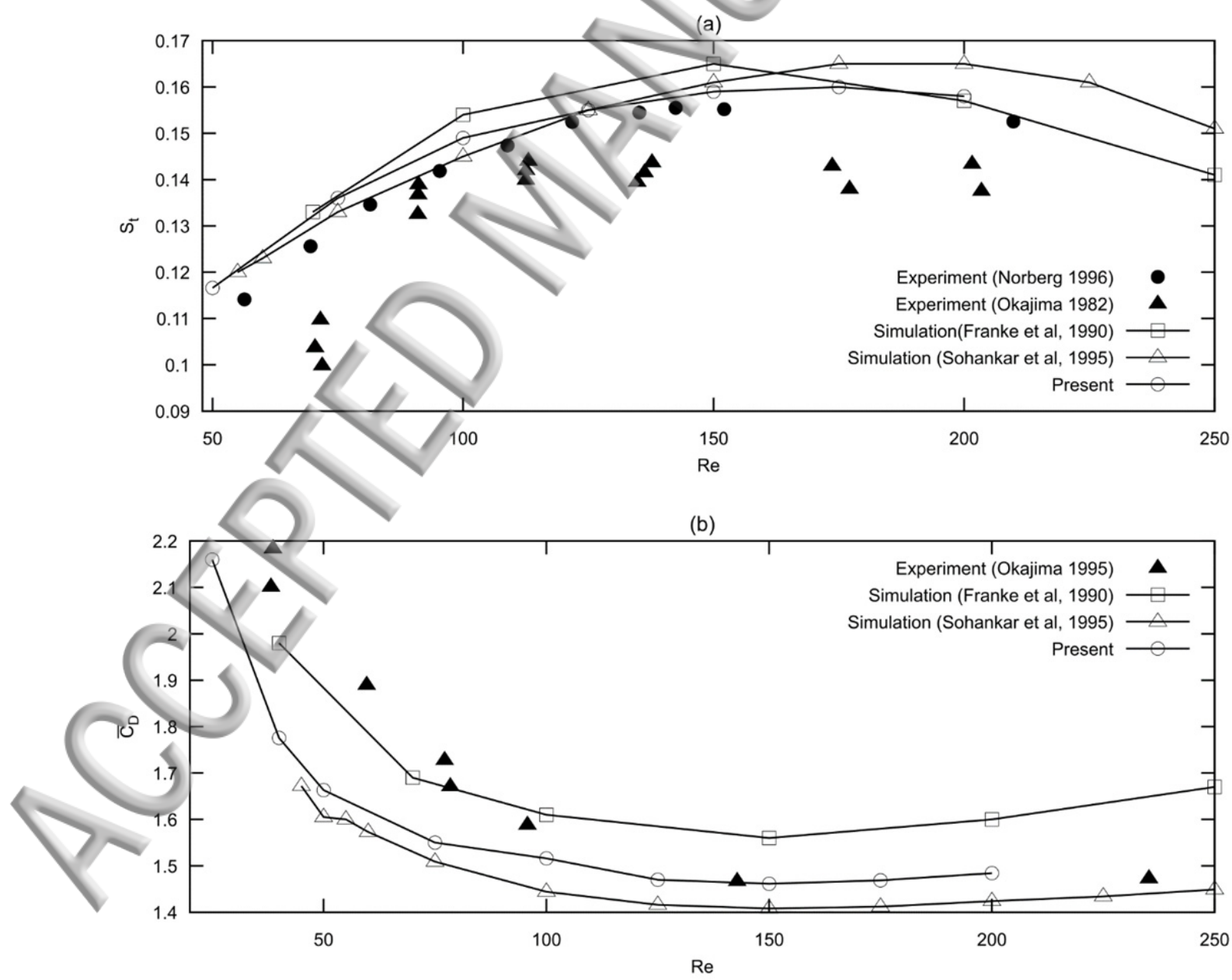
Figure 4 Comparison of simulation results and reported experimental data for single square cylinder at low Reynolds numbers. (a). Strouhal number; (b). Mean drag coefficient.

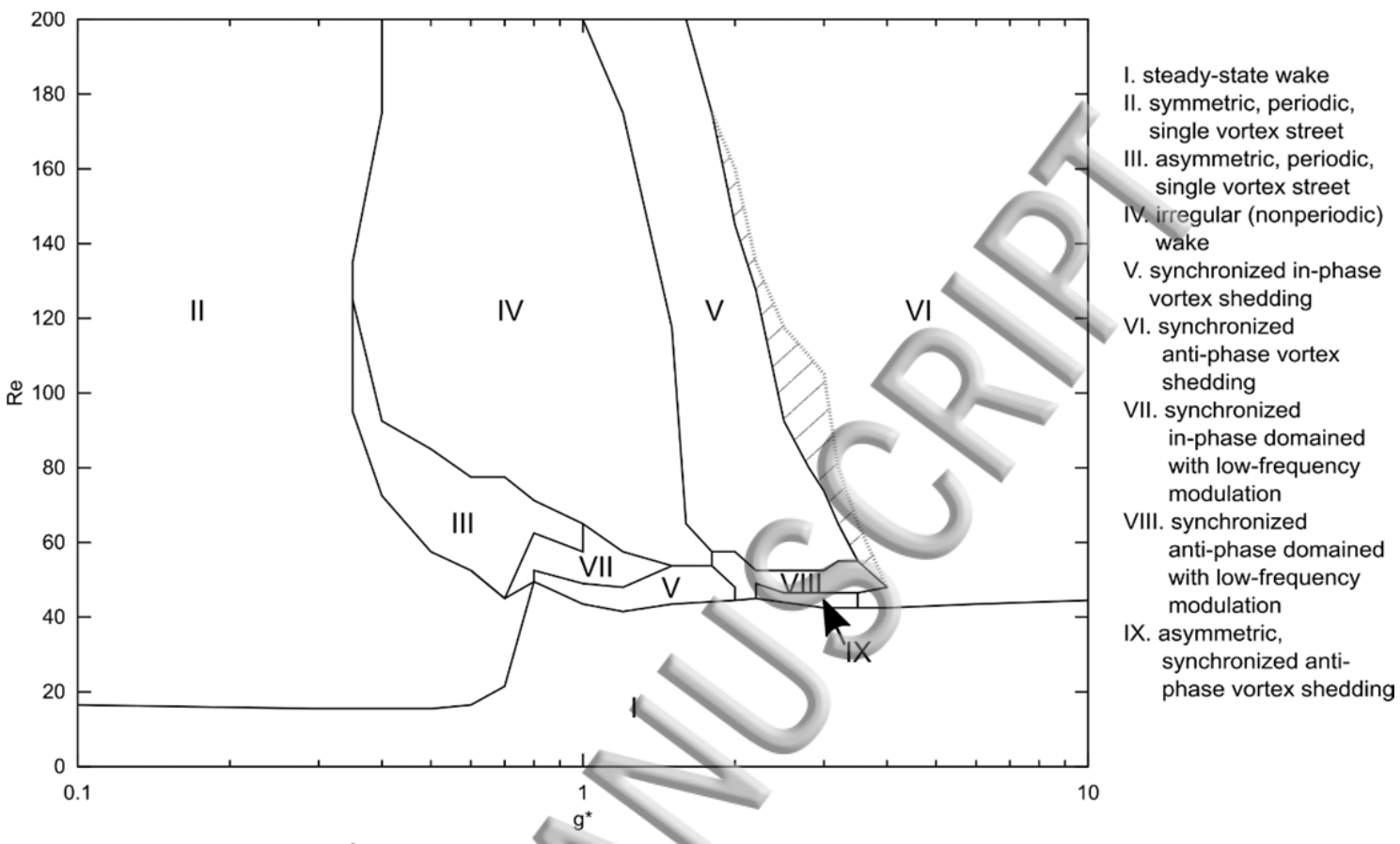

Figure 5. The impact of $\mathrm{g}^{*}$ and $\mathrm{Re}$ on wake patterns and vortex shedding modes 

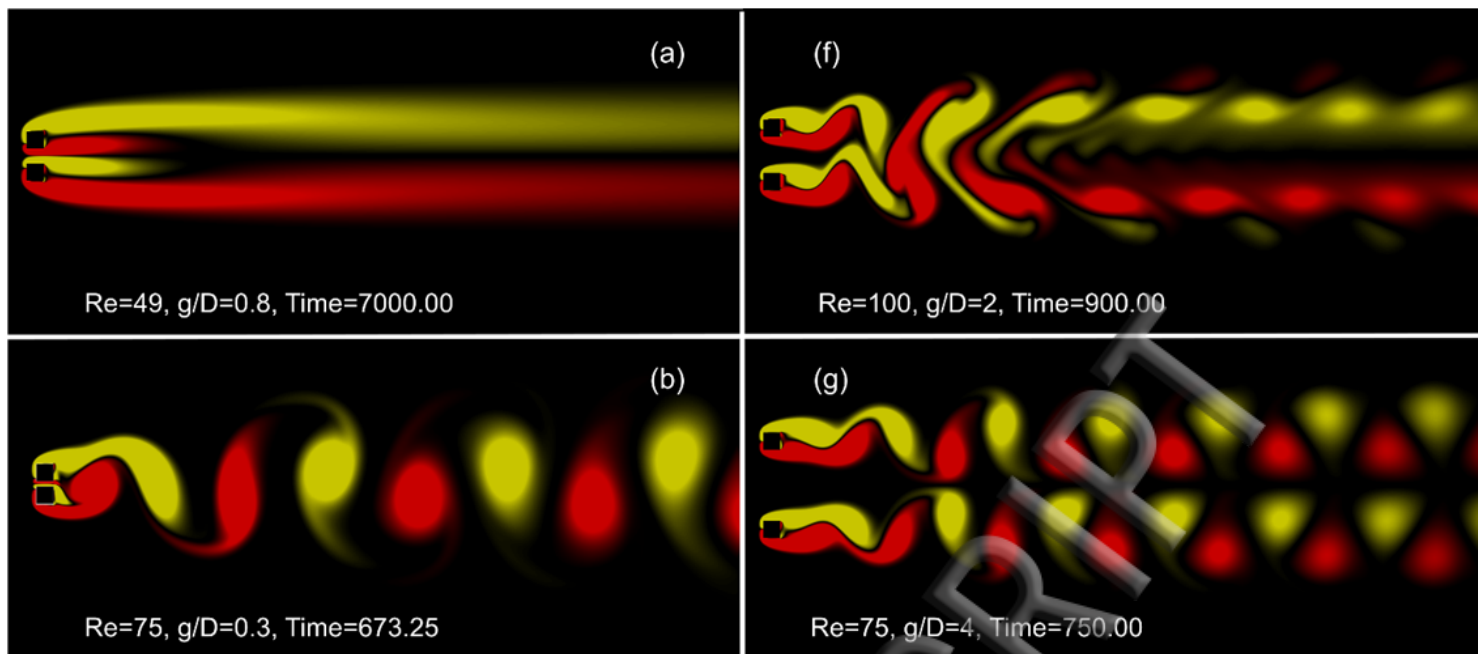

$5, \mathrm{~g} / \mathrm{D}=0.3, \mathrm{Time}=673$
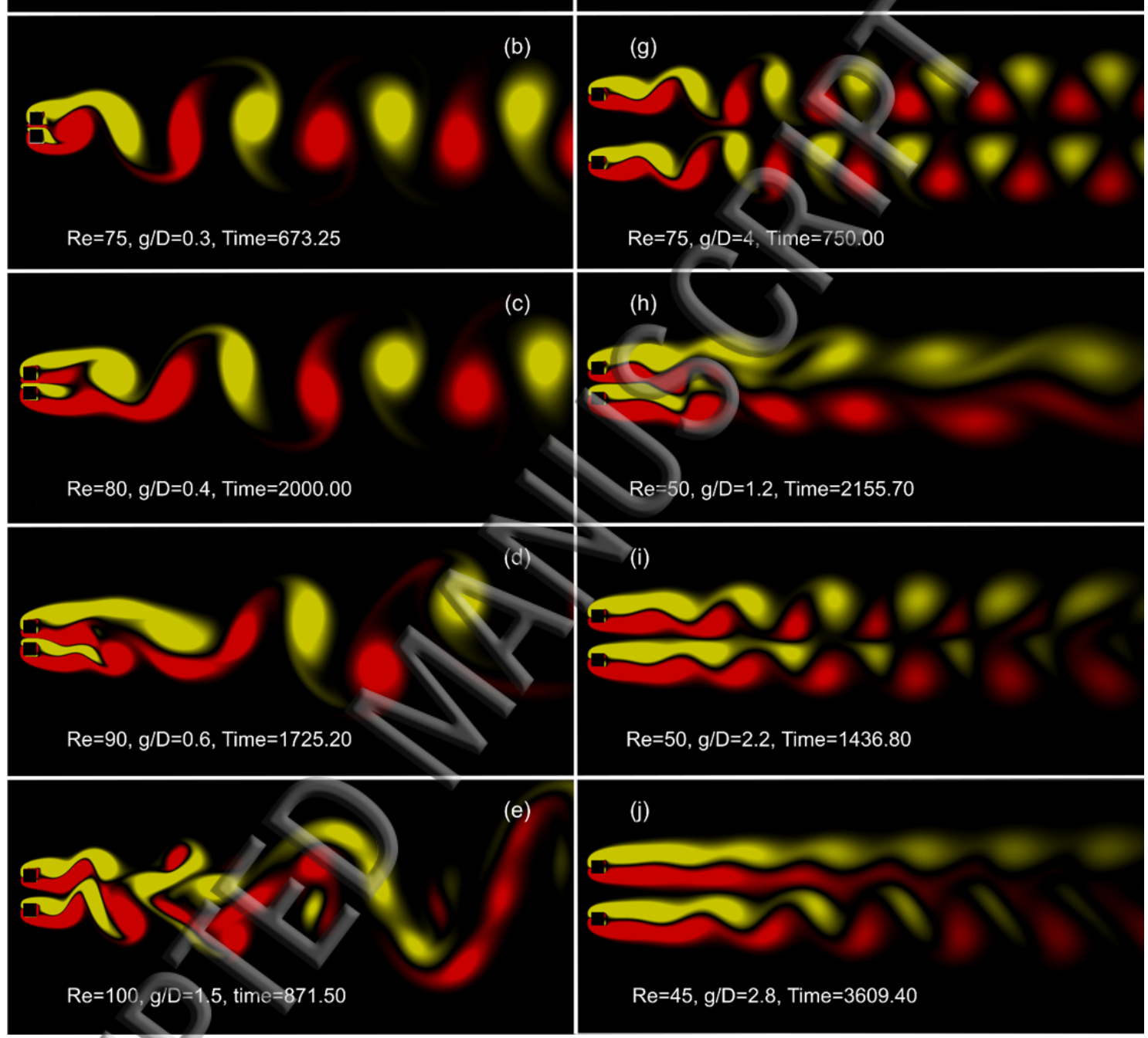

Figure 6. Visualization of instantaneous vorticity field of different wake patterns. (a). steadystate wake (mode I); (b). Symmetric single vortex street (mode II); (c). Asymmetric single vortex street (in this example, the wide wake is formed behind cylinder 1 , and narrow wake behind cylinder 2.) (mode III); (d). Irregular (non-periodic) wake with single vortex street (vortex shedding from freestream side only) (mode IV); (e). Irregular (non-periodic) vortex shedding with two vortex streets in the near wake (vortex shedding from both gap and freestream sides) (mode IV); (f). Synchronized in-phase vortex shedding (mode V); (g). Synchronized anti-phase vortex shedding (mode VI); (h). Synchronized in-phase dominated vortex shedding with low frequency modulation (mode VII); (i). Synchronized anti-phase dominated vortex shedding with low frequency modulation (mode VIII); (j). Asymmetric synchronized anti-phase vortex shedding (mode IX). 

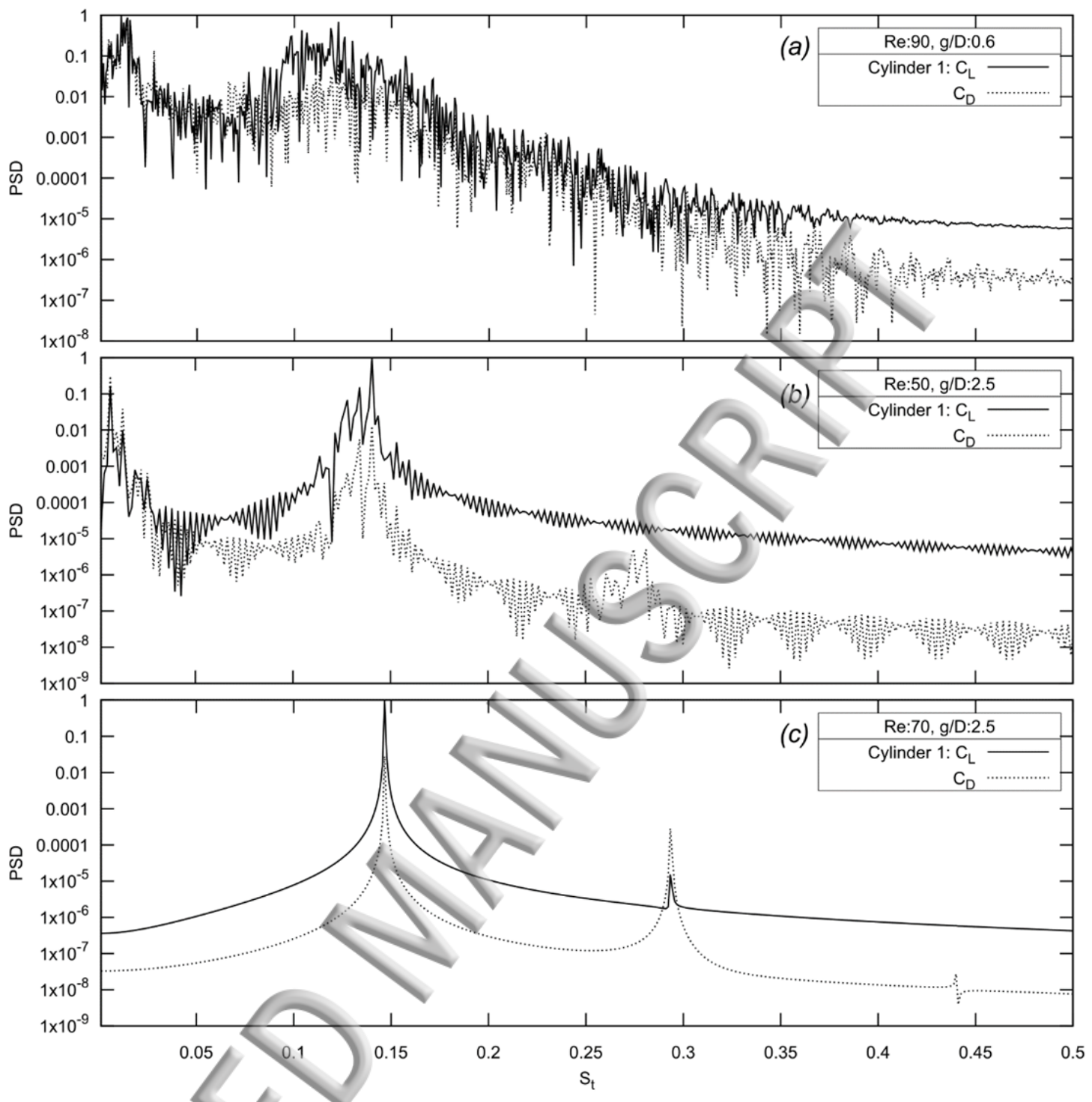

Figure 7. FFT analysis for the time history of lift and drag coefficients of cylinder 1 . Three possible types of shedding frequency are shown in plots of (a), (b) and (c) respectively. The results for cylinder 2 are similar to those for cylinder 1 in all regimes, including the asymmetric regimes. (a). Non-stationary shedding frequency. This corresponds to the irregular vortex shedding regime (mode IV). (b). Multiple frequencies. This corresponds to the regimes with low frequency modulations, namely, in-phase dominated vortex shedding with low frequency modulation (mode VII), and anti-phase dominated vortex shedding with low frequency modulation (mode VIII). (c). Single stationary vortex shedding frequency. This corresponds to all other transient regimes (modes II, III, V, VI and IX). 

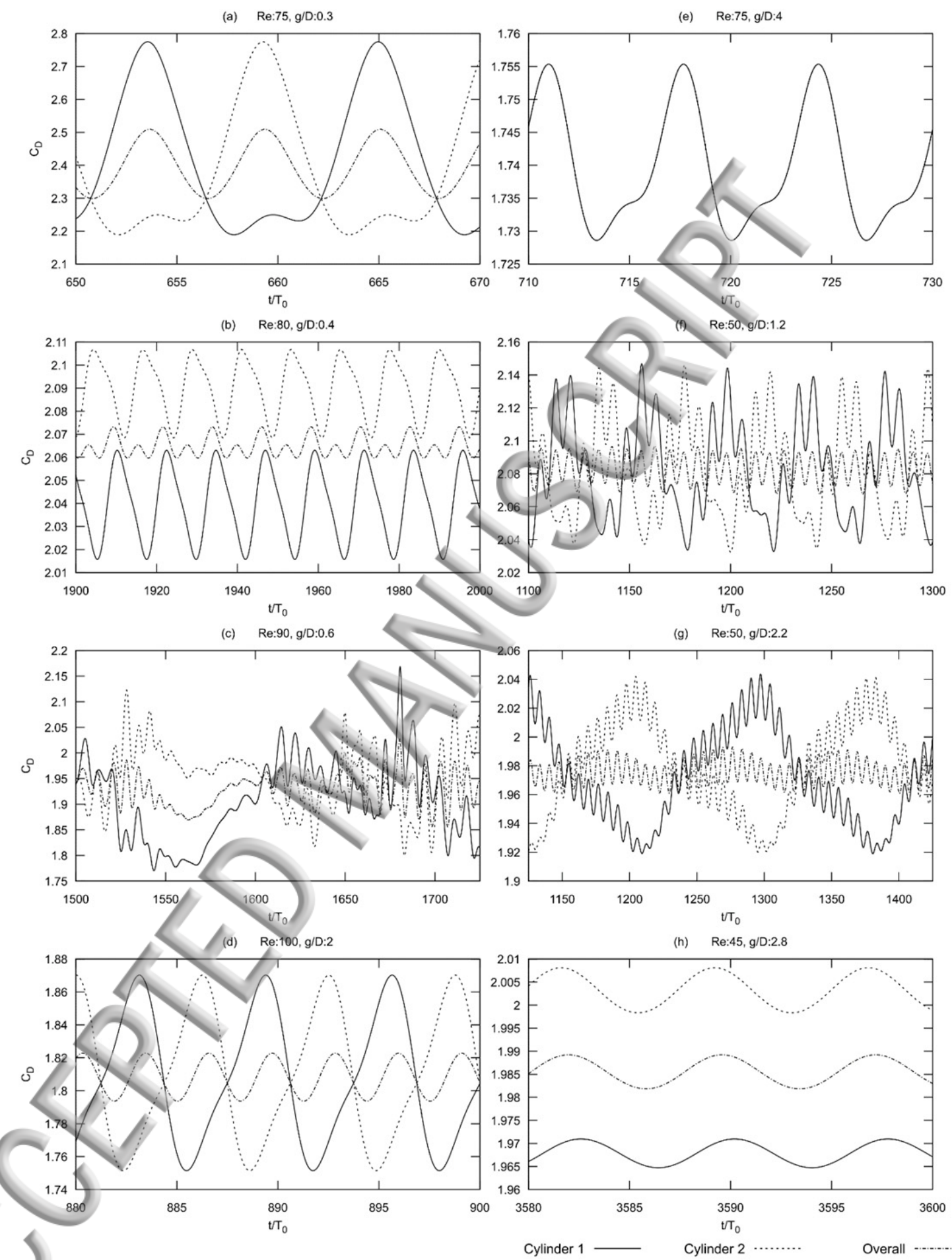

Figure 8. Instantaneous drag coefficient of two cylinders and the instantaneous overall (averaging of cylinders 1 and 2) drag coefficient for different wake modes. (a). Symmetric single vortex street (mode II); (b). Asymmetric single vortex street (mode III); (c). Irregular vortex shedding (mode IV); (d). Synchronized in-phase vortex shedding (mode V); (e). Antiphase vortex shedding (mode $\mathrm{VI}$ ); (f). Synchronized in-phase dominated vortex shedding with low frequency modulation (mode VII); (g). Synchronized anti-phase dominated vortex 
shedding with low frequency modulation (mode VIII); (h). Asymmetric synchronized antiphase vortex shedding (mode IX)

(a) $\quad \operatorname{Re}: 75, g / D: 0.3$

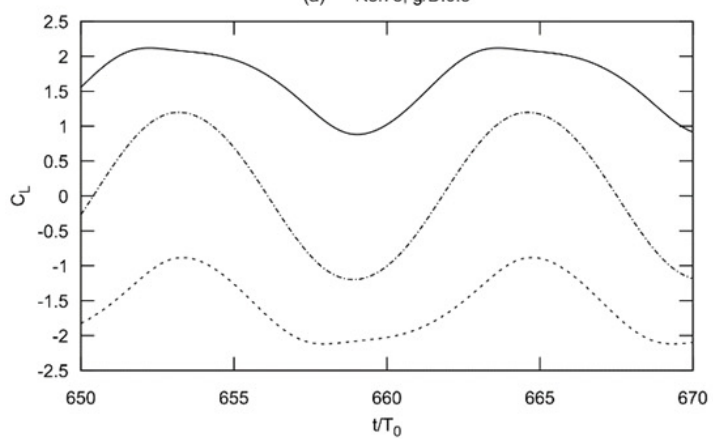

(b) Re:80, g/D:0.4

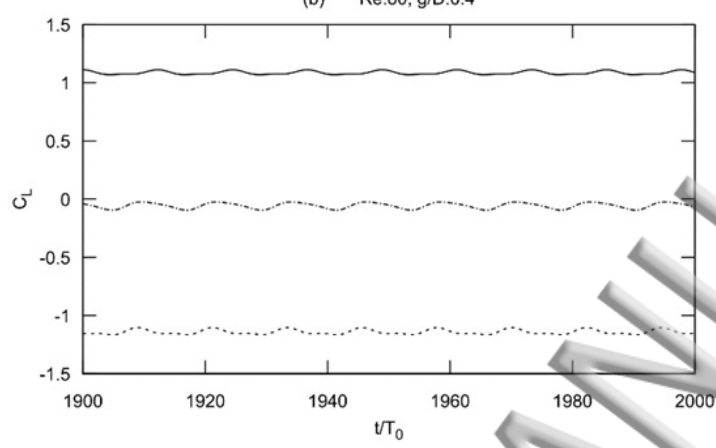

(c) Re:90, g/D:0.6

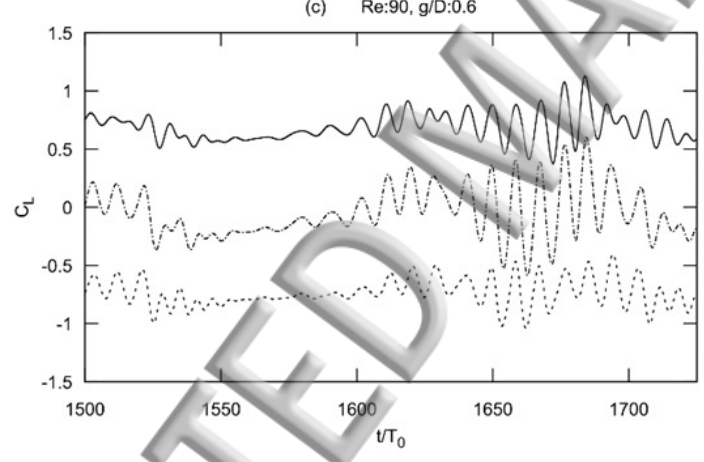

(d) Re:100, g/D:2

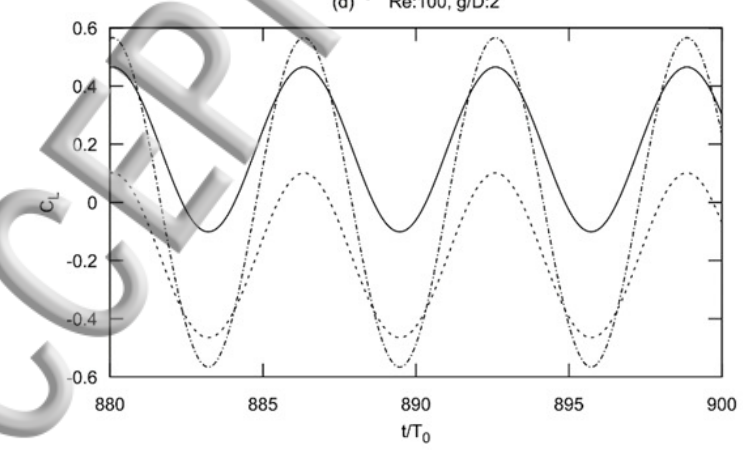

(e) $\quad \operatorname{Re}: 75, g / D: 4$
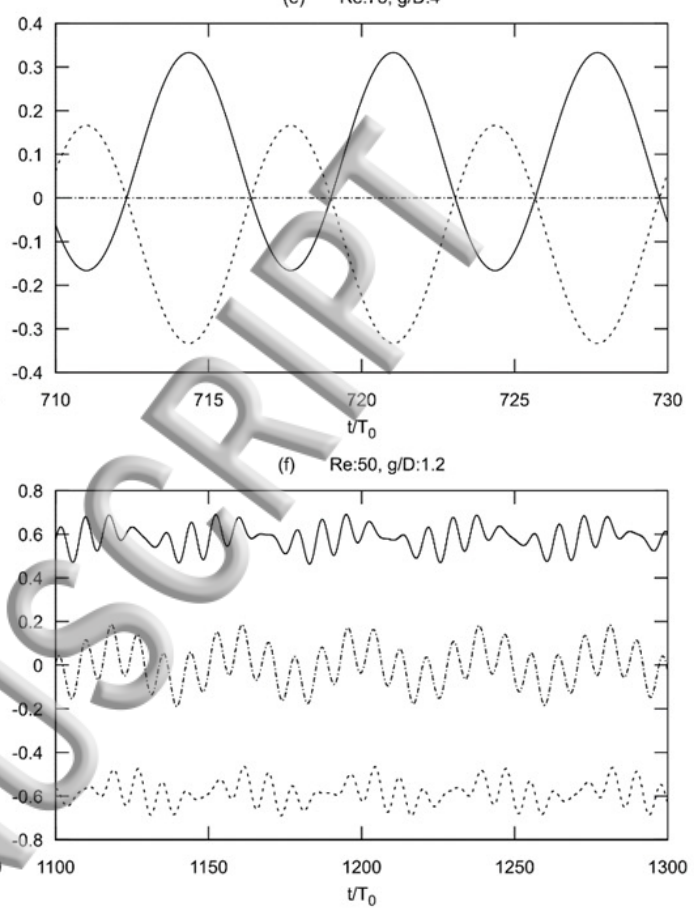

(g) Re:50, g/D:2.2

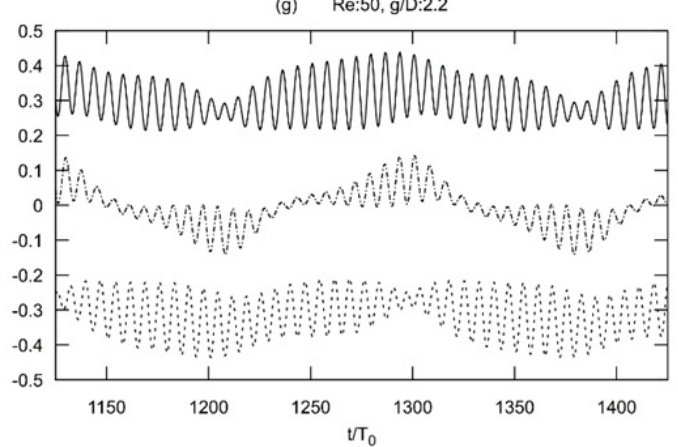

(h) Re:45, g/D:2.8

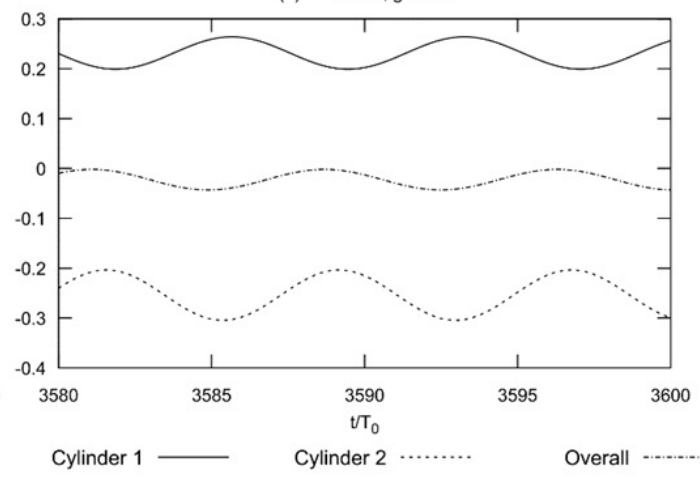

Figure 9. Instantaneous lift coefficient of two cylinders and the instantaneous overall (summation of cylinders 1 and 2) lift coefficient for different wake modes. (a) mode II; (b) mode III; (c) mode IV; (d) mode V; (e) mode VI; (f) mode VII; (g) mode VIII; (h) mode IX. 

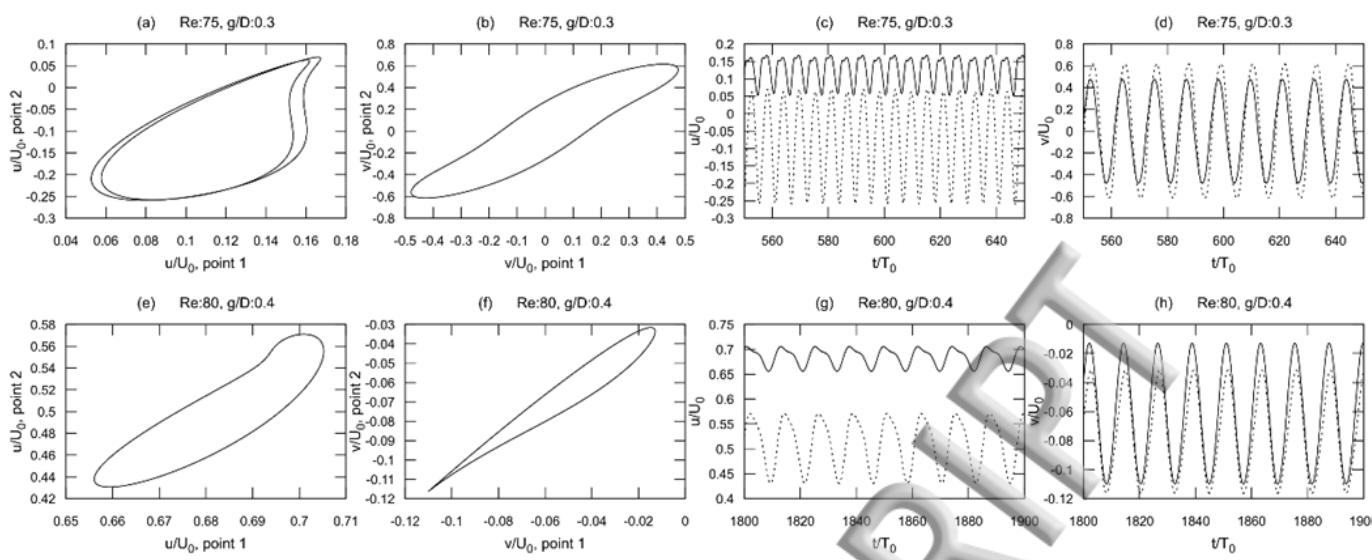

(h) Re:80, g/D:0.4
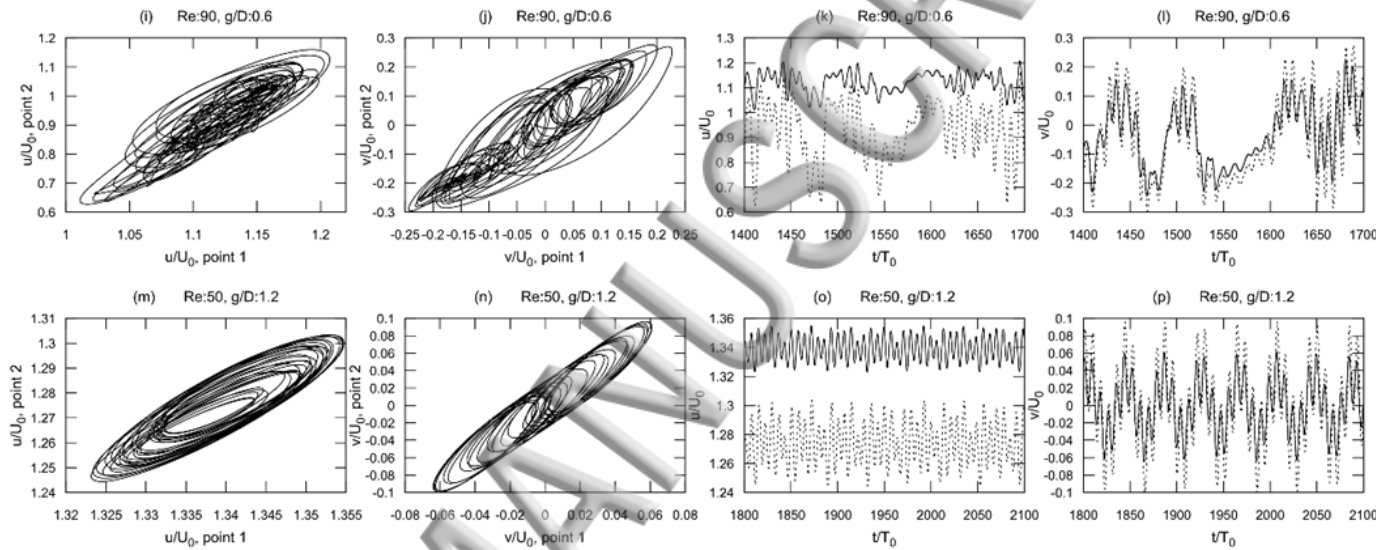

(n) Re:50, g/D:1.2
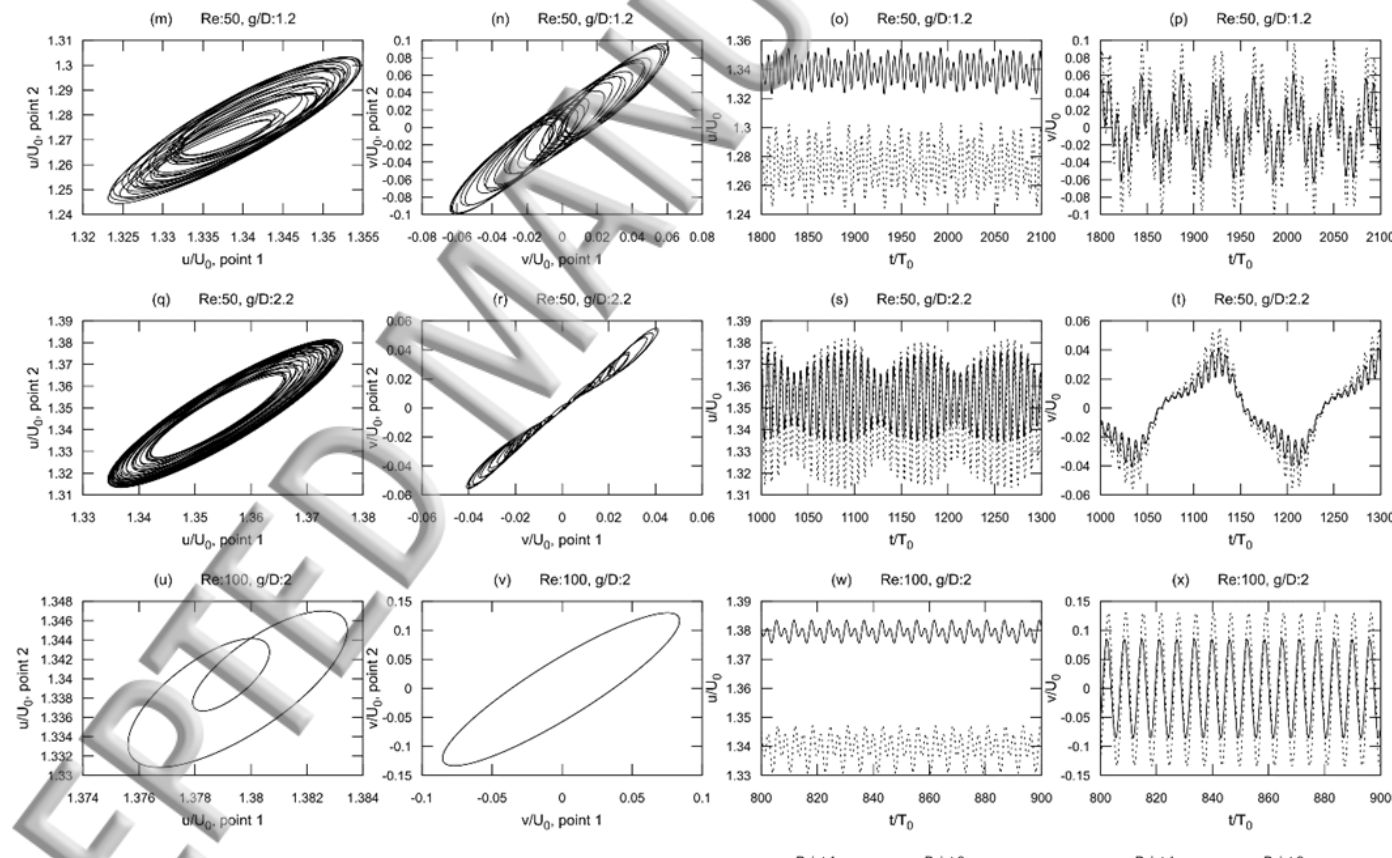

Figure 10. Instantaneous velocity in two points $(x / D=1, y / D=0)$ and $(x / D=1.5, y / D=0)$ in the wake. The correlation of $u / \bigcup_{0}$ in these two points, $v / U_{0}$ in two points and time history of instantaneous $u$ and $v$ are shown for different modes respectively. (a)-(d). Single vortex street mode (mode II); (e)-(h). Asymmetric single vortex street mode (mode III). Asymmetric anti-phase mode (mode IX) usually has similar correlation plot for $u$ and $v$. (i)-(I). Irregular shedding mode (mode IV); $(\mathrm{m})$-(p). Synchronized in-phase dominated vortex shedding with low frequency modulation (mode VII); (q)-(t). Synchronized anti-phase dominated vortex shedding with low frequency modulation (mode VIII); (u)-(x). Synchronized in-phase mode (mode V). Synchronized anti-phase mode (mode VI) shares a similar plot. 


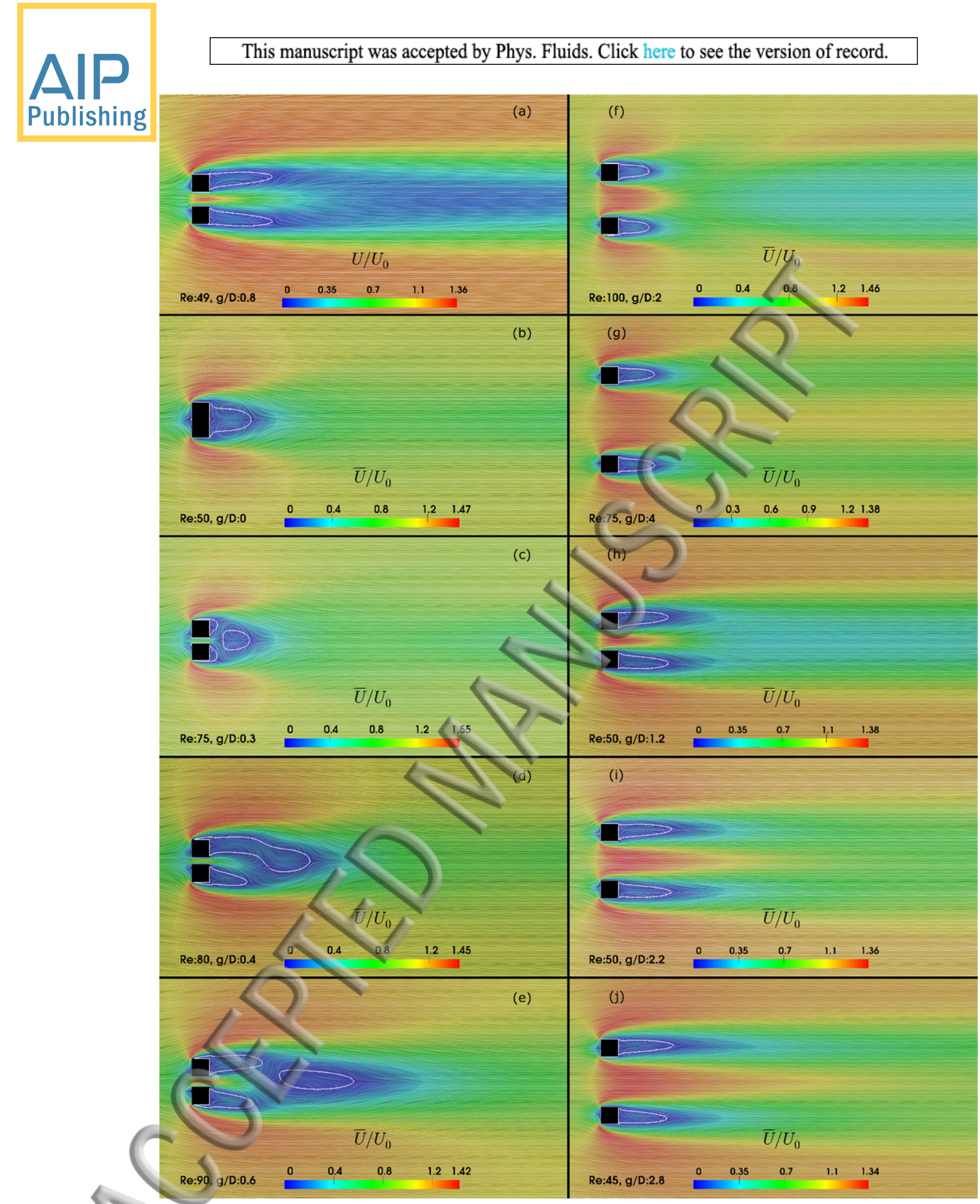

Figure 11. Mean flow field rendered with surface line integral convolution (LIC) and colored by the magnitude of mean velocity. White lines in plots are cylinder surface and isoline of $\overline{U_{x}}=0$ illustrating the recirculation regions. (a). Steady-state wake (mode I); (b). $\mathrm{g}^{*}=0$ and $\mathrm{Re}=50$ (based on D) (mode II); (c). Symmetric single vortex street (mode II); (d). Asymmetric single vortex street (mode III); (e). Irregular vortex shedding (mode IV); (f). Synchronized in- 
phase vortex shedding (mode V); (g). Synchronized anti-phase vortex shedding (mode VI). (h). In-phase dominated vortex shedding with low frequency modulation (mode VII); (i). Antiphase dominated vortex shedding with low frequency modulation (mode VIII); (j). Asymmetric synchronized anti-phase vortex shedding (mode IX).

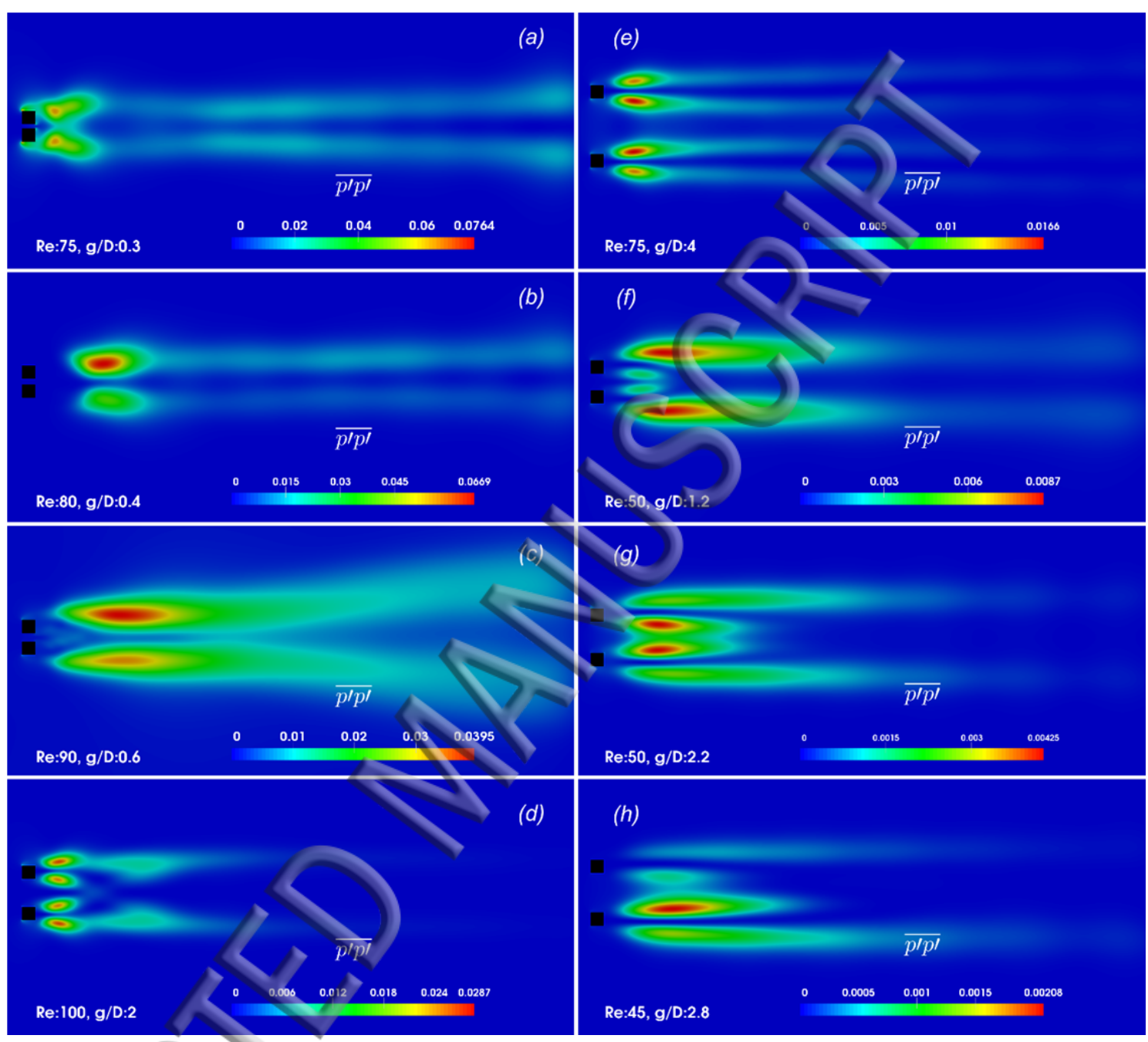

Figure 12. Pressure fluctuations $\overline{p^{\prime 2}} /\left(\rho^{2} U_{0}^{4}\right)$ of different vortex shedding modes. (a) mode II; (b) mode III; (c) mode IV; (d) mode V; (e) mode VI; (f) mode VII; (g) mode VIII; (h) mode IX. 

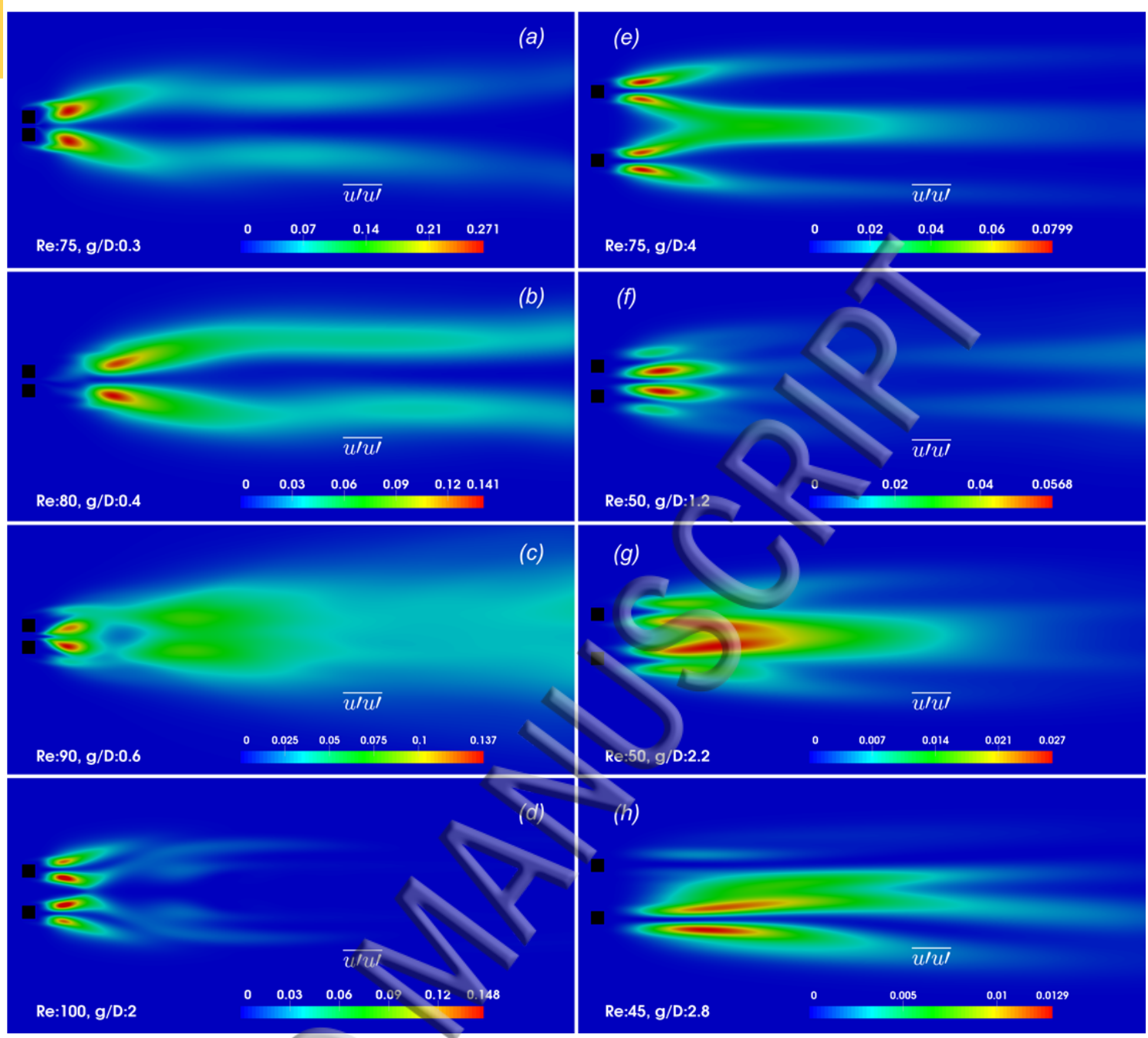

Figure 13. X-component velocity fluctuations $\left(\overline{u^{\prime 2}} / U_{0}^{2}\right)$ of different vortex shedding modes. (a) mode II; (b) mode III; (c) mode IV; (d) mode V; (e) mode VI; (f) mode VII; (g) mode VIII; (h) mode IX. 

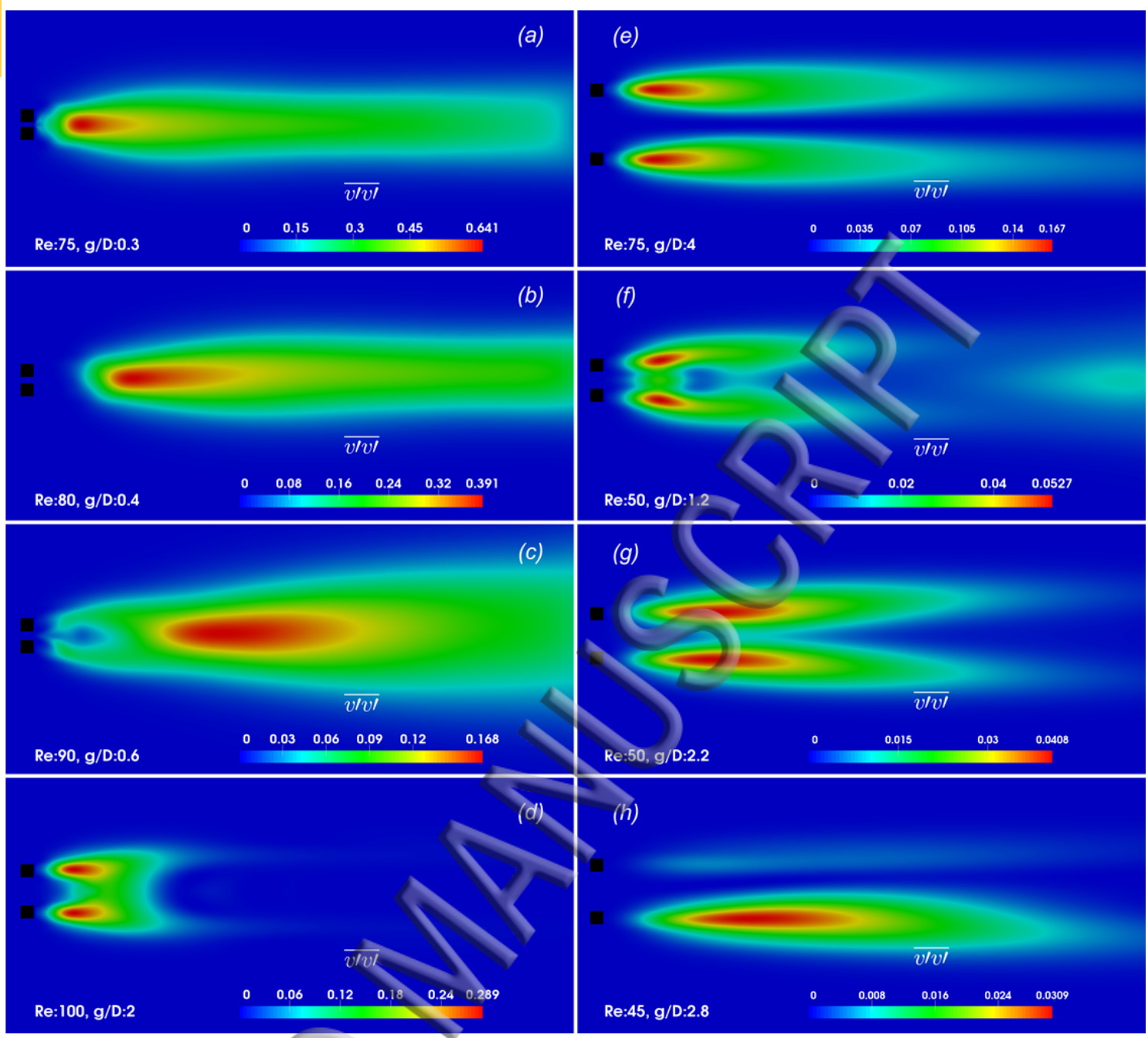

Figure 14. Y-component velocity fluctuations $\left(\overline{v^{\prime 2}} / U_{0}^{2}\right)$ of different vortex shedding modes. (a) mode II; (b) mode III; (c) mode IV; (d) mode V; (e) mode VI; (f) mode VII; (g) mode VIII; (h) mode IX. 


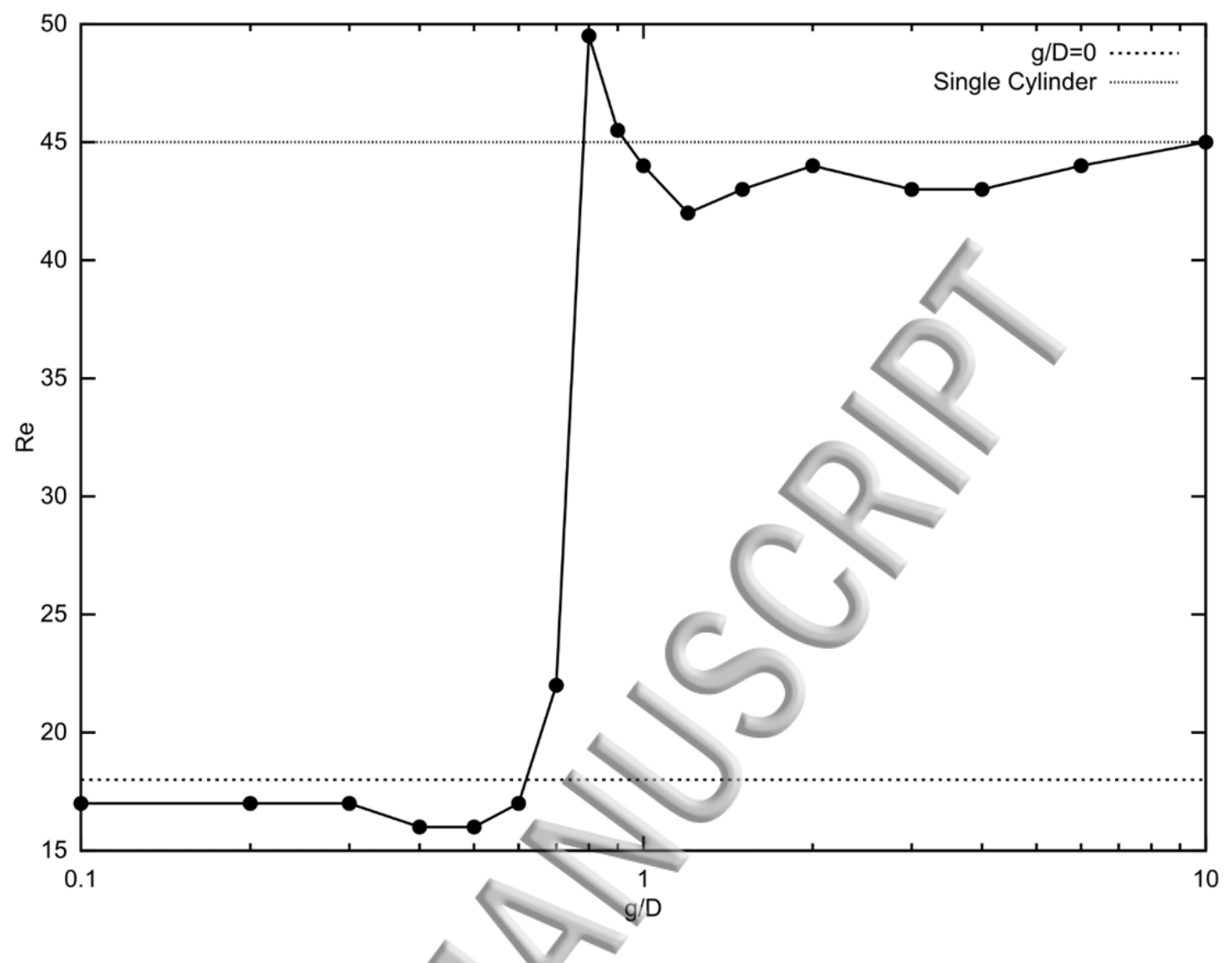

Figure 15. Comparison of critical Reynolds number at different gap ratios. 


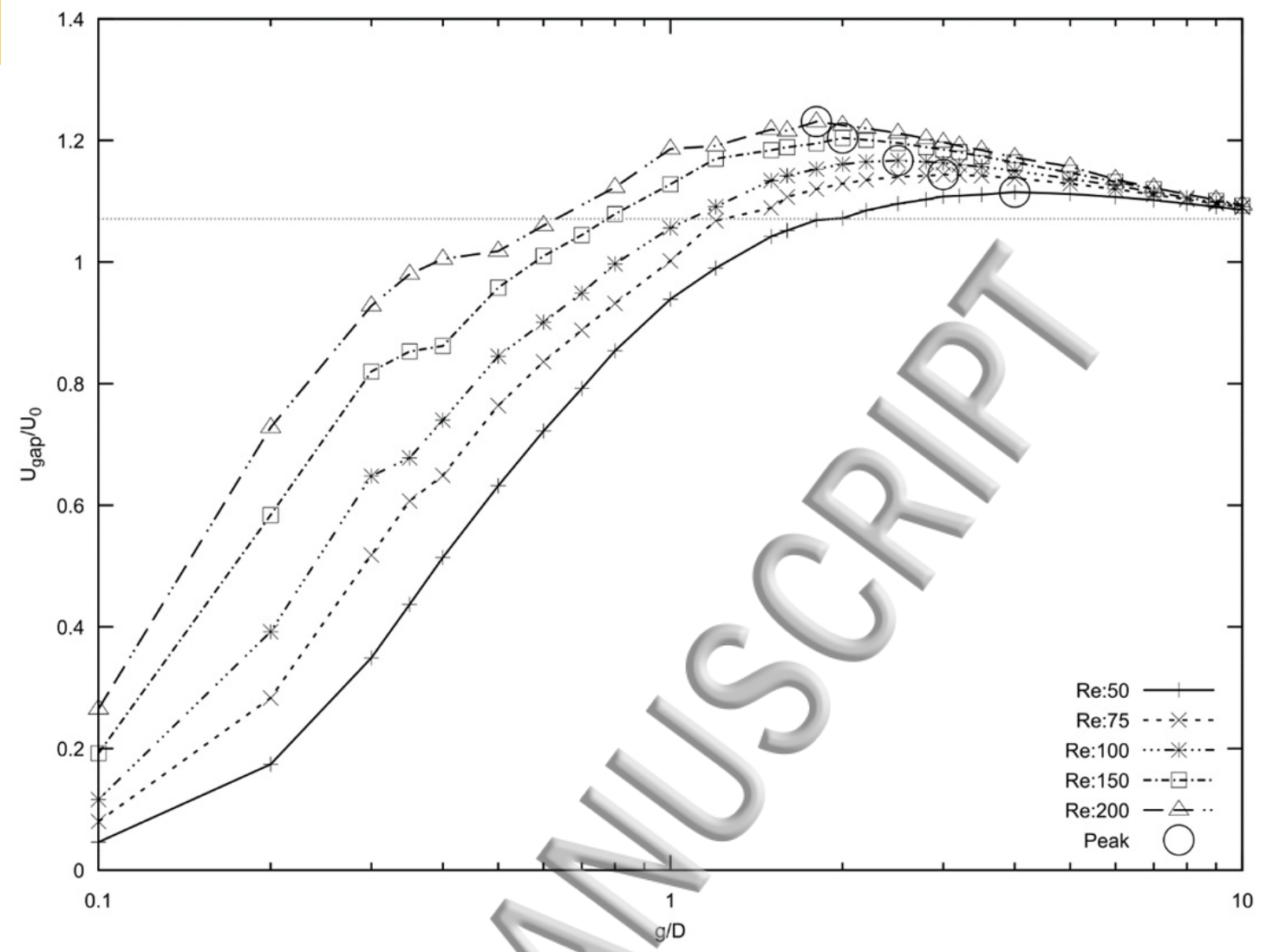

Figure 16. Mean gap flow variation with $\mathrm{g}^{*}$ and $\mathrm{Re}$ 

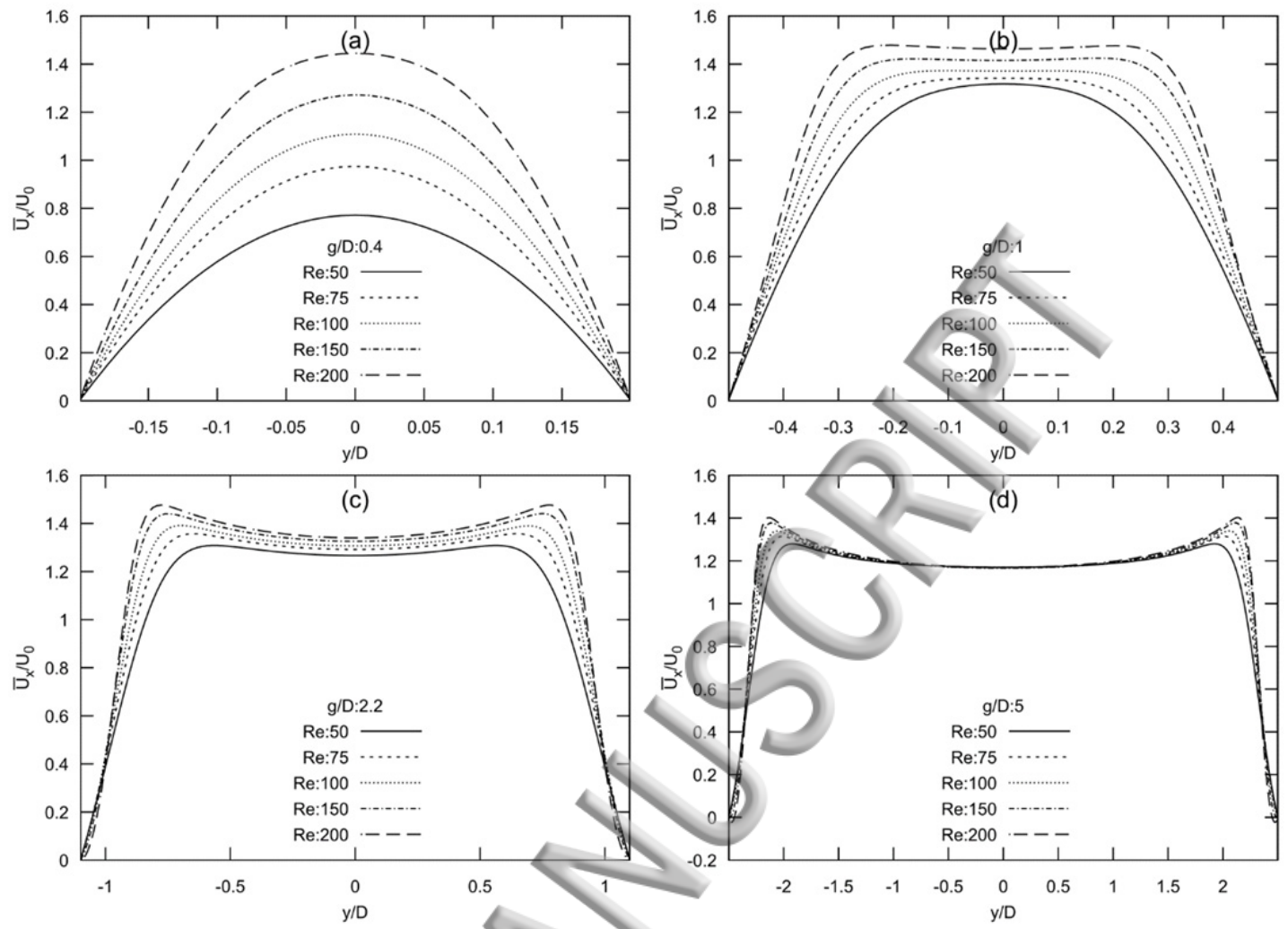

Figure 17. X-component mean flow profile in the gap (between the two cylinders) at $x=0$. (a) $g^{*}=0.4 ;$ (b) $g^{*}=1$; (c) $g^{*}=2.2$; (d) $g^{*}=5$.
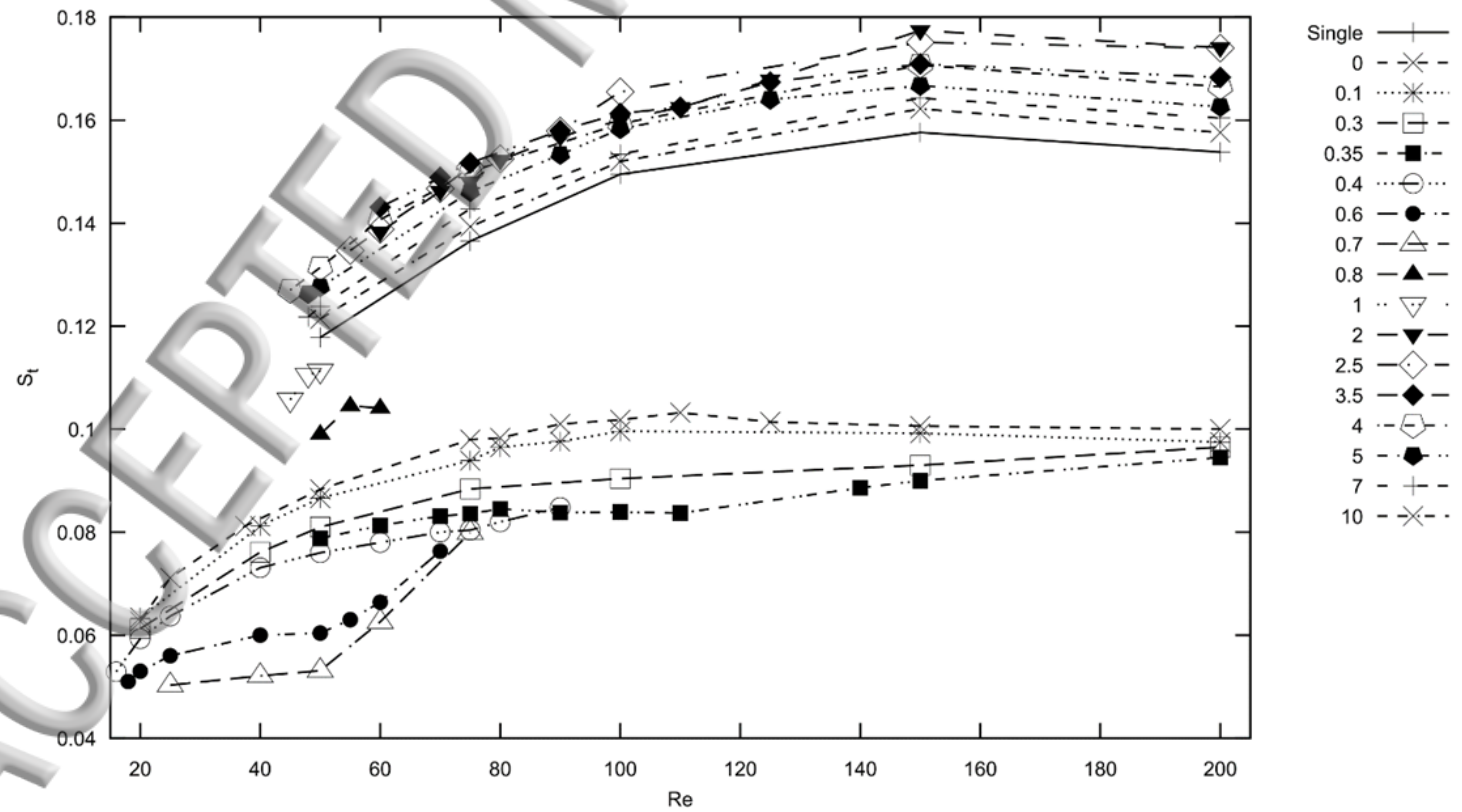

Figure 18. Impact of gap ratio and Reynolds number on Strouhal number 


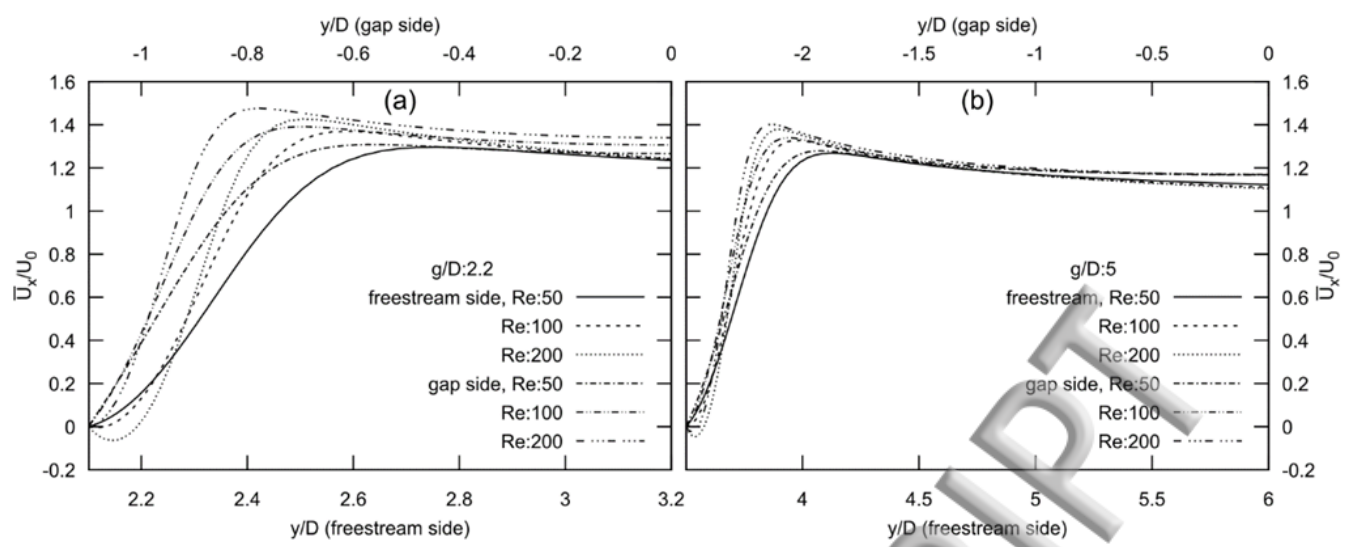

Figure 19. Near-wall mean velocity (x-component) profiles in gap side and freestream side at $x=0$. Gap side is from upper surface of cylinder $2\left(y / D=-0.5 g^{*}\right)$ to the gap center $(y / D=0)$. Freestream side is from upper surface of cylinder $1\left(y / D=1+0.5 g^{*}\right)$ to $y / D=1+g^{*}$. (a) $g^{*}=2.2$; (b) $g^{*}=5$.
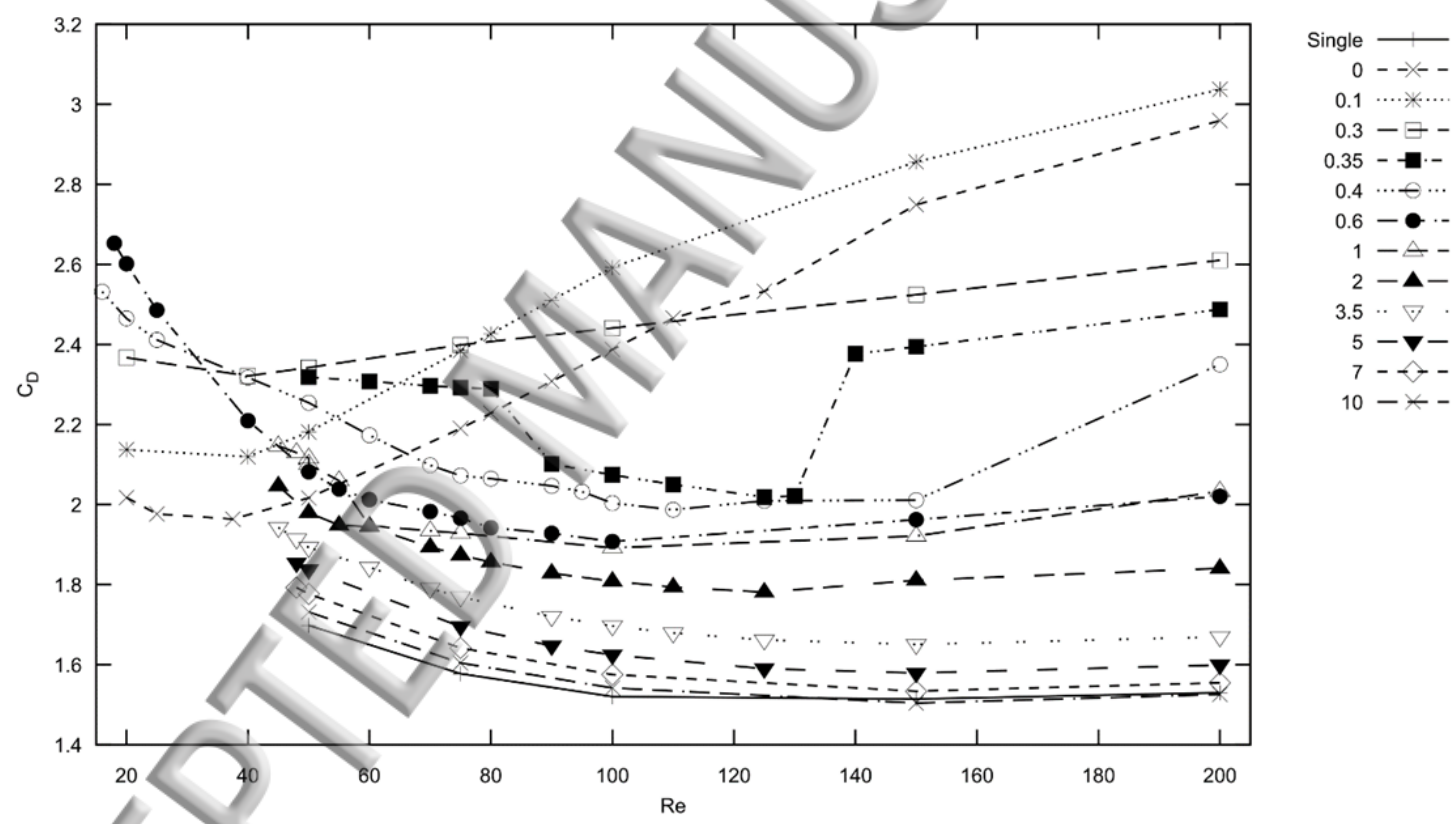

Figure 20. Impact of gap ratio and Reynolds number on mean drag coefficient 


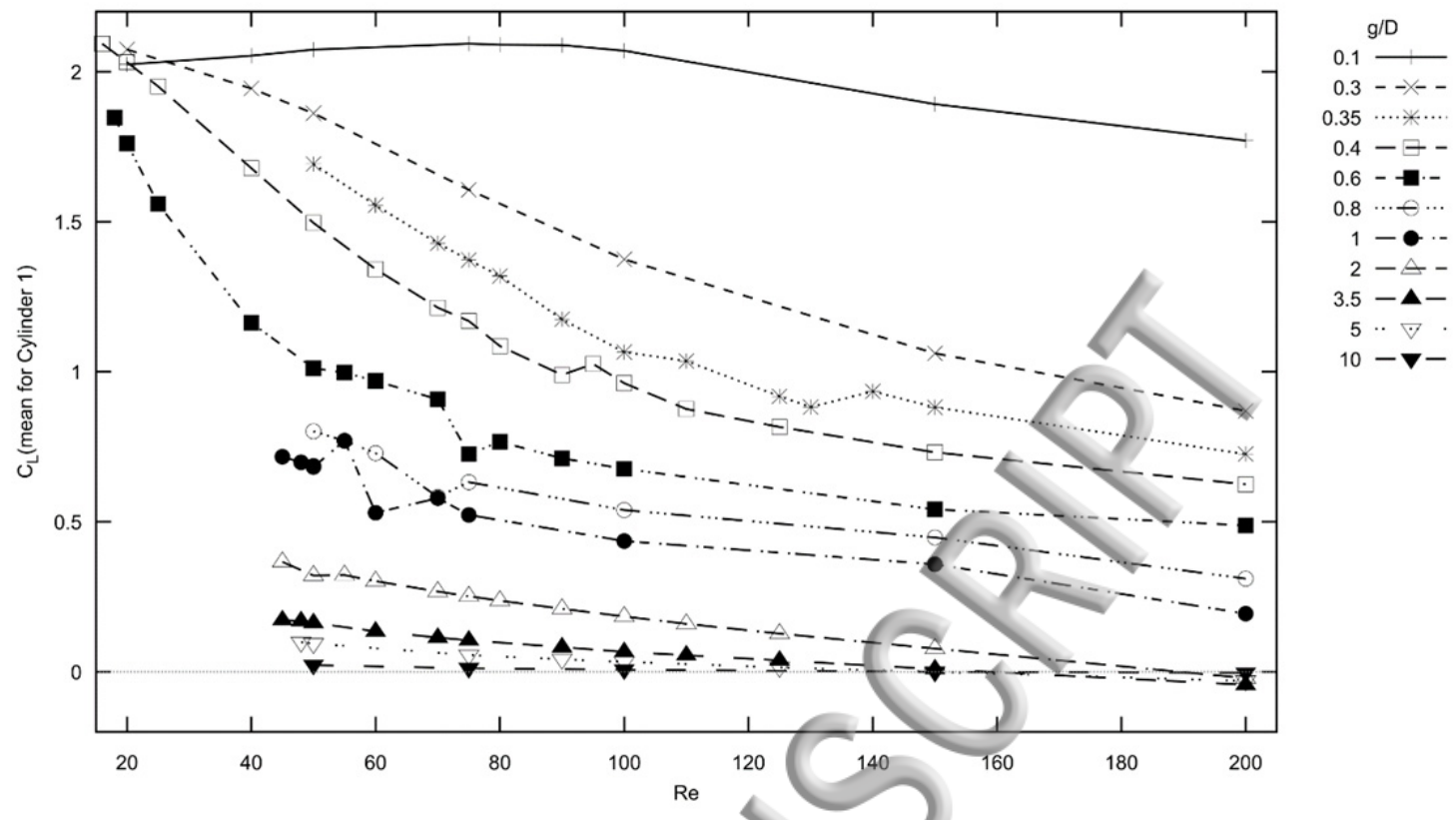

Figure 21. Impact of gap ratio and Reynolds number on mean lift force 